\title{
ROLES OF RETINOIC ACID IN GERM CELL DIFFERENTIATION
}

Marius TELETIN a,b,c,d,e, Nadège VERNET a,b,c,d, Norbert B. GHYSELINCK a,b,c,d and Manuel MARK a,b,c,d,e.

a) Institut de Génétique et de Biologie Moléculaire et Cellulaire (IGBMC), Department of Functional Genomics and Cancer, 1 rue Laurent Fries, BP10142, F-67404, Illkirch, France

b) Centre National de la Recherche Scientifique (CNRS), UMR7104, France

c) Institut National de la Santé et de la Recherche Médicale (INSERM), U964, France

d) Université de Strasbourg (UNISTRA), France

e) Hôpitaux Universitaires de Strasbourg (HUS), France

\section{Contents}

1. Introduction

2. ATRA signaling and gonocytes in the fetal gonads

2.1. CYP26B1 acts as a MPS for male gonocytes

2.2. CYP26B1 prevents proliferation as well as death of male gonocytes

2.3. ATRA is required for the production of "differentiating" spermatogonia from male gonocytes

2.4. ATRA possibly acts as a MIS for female gonocytes

2.5. Stra8 as the meiotic gatekeeper

2.6. An alternative viewpoint of meiosis induction in female gonocytes

3. ATRA signaling is instrumental to spermatogonia differentiation in the prepubertal and adult testis

3.1. The transition from undifferentiated to differentiating spermatogonia critically relies on ATRA

3.2. The sources of ATRA destined for spermatogenesis are intrinsic to the SE

4. ATRA is instrumental to meiosis in spermatocytes

5. ATRA metabolism within the se controls the timing and spatial patterning of spermatogonia differentiation

5.1. The SE cycle and wave both rely on retinoid signaling

5.2. Endogenous ATRA levels in the SE are tightly regulated

5.3. RALDH activity regulates the spermatogenic wave

6. Male GC are both direct and remote targets of ATRA action: lessons from mouse mutants lacking retinoid receptors

6.1. RAR in fetal GC differentiation

6.2. RARG controls the capacity of spermatogonia to respond to ATRA

6.3. The response of spermatogonia to ATRA relies on RARG/RXR heterodimers

6.4. RARA in Sertoli cells also contribute to ATRA functions in the SE

7. Concluding remarks

References 


\section{INTRODUCTION}

Germ cells $(\mathrm{GC})$ are unique in their capacity to divide meiotically and to halve their genetic material, generating haploid gametes, oocytes in the female and spermatozoa in the male, that transfer the genetic information from one generation to the next. Due to ethical issues restricting access to human material for research purposes, studies documenting the fate of human GC are sparse and much of what is known about gamete formation has been obtained from the mouse. However, the characteristic cellular events and chronology of the key steps of gametogenesis are similar in mice and humans.

The first GC population established during development, primordial GC (PGC) are segregated from the somatic cell lineage (epiblast) at early gastrulation, around embryonic day (ED) 6.25 in the mouse. PGC proliferate while migrating along the hindgut endoderm to reach the embryonic gonads during the late embryonic period (i.e., ED10.5-ED11.0) (reviewed in McLaren \& Lawson, 2005). Once in the gonad and now known as gonocytes (Clermont \& Perey, 1957), GC follow a sexually dimorphic pathway dictated by cues from the somatic cells (Fig. 1). In the fetal ovary, female gonocytes initiate prophase I of meiosis at about ED13.5. As primary oocytes, they pass through the leptotene, zygotene and pachytene stages before arresting after birth in diplotene, surrounded by a single layer of somatic, follicle cells. Upon completion of puberty, several primary oocytes achieve meiosis I and then divide, yielding haploid secondary oocytes at each menstrual cycle (Fig. 1). Progression through meiosis in oocytes pauses again in metaphase II, shortly before ovulation, to complete the second meiotic division after fertilization in the oviduct. In the fetal testis, male gonocytes, surrounded by somatic cells of the testis cords, undergo a mitotic arrest in G0/G1 (Fig. 1). After birth, they resume proliferation and undergo a transition to spermatogonia. Meiosis in primary spermatocytes begins during the prepubertal period, by the end of the first post-natal week (PNW). The two meiotic divisions proceed without interruption, leading to the formation of haploid secondary spermatocytes, then of round spermatids. The latter undergo morphological changes to finally become spermatozoa (Fig. 1). Spermatogenesis, the process yielding spermatozoa from spermatogonia, is completed for the first time at PNW5 and continues throughout adulthood. It takes place in the seminiferous epithelium of the testis, under the control of a somatic, supporting, cell-type called the Sertoli cell (reviewed in Bowles \& Koopman, 2007; Kocer, Reichmann, Best, \& Adams, 2009; Griswold, 2016).

Spermatogenesis is critically dependent upon vitamin A (vitA), acting through its metabolite, all-trans retinoic acid (ATRA). This has been clear since the pioneering study by Wolbach \& Howe (1925) showing that spermatogenesis fails in rats fed a vitAdeficient diet, and the subsequent demonstration that it can be successfully restored upon systemic administration of ATRA. Then, several genetic and pharmacological studies have highlighted the importance of ATRA in regulating meiosis.

The canonical pathway for ATRA signaling has been elucidated over the years, mainly though gene targeting studies in the mouse (Fig. 2). Conversion of vitA to ATRA requires two sequential oxidative steps. The first one is catalyzed by either cytosolic alcohol dehydrogenases or microsomal retinol dehydrogenases $(\mathrm{RDH})$ to generate retinaldehyde (RAL). The second step, generating ATRA, is catalyzed by RAL dehydrogenases (RALDH1, RALDH2, and RALDH3, encoded by the Aldh1a1, Aldh1a2 and Aldh1a3 genes, respectively). In cells, ATRA binds to and activates 
nuclear receptors (RARA, RARB and RARG), which are ligand-dependent transcriptional regulators. They usually function in the form of heterodimers with rexinoid receptors (RXRA, RXRB and $R X R G$ ) to control expression of ATRA-target genes through binding to retinoic acid response elements (RARE) located in genomic regulatory regions. Aside from synthesis, degradation of ATRA is also an important mechanism that protects cells from inadequate ATRA stimulation. It is catalyzed by three cytochrome P450 hydroxylases (CYP26A1, CYP26B1 and CYP26C1), which hydroxylate ATRA into water-soluble products that are less active and readily excretable (reviewed in Duester, 2008; Samarut \& Rochette-Egly, 2012).

In this review, we discuss our current understanding on how ATRA regulates GC differentiation and entry into meiosis. We also provide complementary information and some novel details as to the sources and the functions of ATRA in these processes.

\section{ATRA SIGNALING AND GONOCYTES IN THE FETAL GONADS}

The timing of meiosis entry in mammals is strikingly different in males and females (Fig. 1). It has been hypothesized that the decision of female GC to enter meiosis is an intrinsic property (i.e., a GC autonomous event, for instance timed by cell divisions), with no reference to somatic cues: they spontaneously enter meiosis at ED13.5 unless prevented from doing so by a putative meiosis preventing substance (MPS). The fact that disaggregated ED11.5 male GC enter meiosis when transferred to lung cell aggregates favors the existence of a MPS synthesized by somatic cells of the fetal testis and acting on GC at a short distance. An alternative hypothesis proposes that GC enter meiosis under the influence of a somatic, diffusible, meiosis inducing substance (MIS), whose existence in the fetal mouse ovary, but not testis, was deduced from co-culture experiments. Such a MIS has to be also present in nongonadal sites, because GC that fail to reach the gonad but instead migrate to ectopic sites as, for instance, the mesonephros, enter meiosis irrespective of their genetic sex. It is worth noting here that the MPS hypothesis can also account for the fact that male gonocytes enter meiosis in non-gonadal sites, where they escape the inhibitory influence of MPS. Whether meiosis entry requires a female MIS only, a male MPS only, or both a MIS and a MPS is still a matter of debate, even though the latter option represents the prevailing model (reviewed in Bowles \& Koopman, 2007; Kocer, Reichmann, Best, \& Adams, 2009).

\subsection{CYP26B1 acts as a MPS for male gonocytes}

The possible role for endogenous ATRA in mouse GC differentiation was initially raised by the sexually dimorphic expression of Cyp26b1, which is expressed in the embryonic gonads of both sexes at ED11.5, but becomes male-specific by ED12.5 with strong expression in the somatic cells of the testis (Bowles et al., 2006; Fig. 3). Then, pharmacological studies have shown that exposure of embryonic testes in organ culture to exogenous ATRA or to the CYP inhibitor ketoconazole induces (i) aberrant expression of meiotic markers such as Stra8 (encoding a meiotic gatekeeper, see below), Dmc1 (encoding a meiotic recombinase) and Sycp3 (encoding a synaptonemal complex protein) and (ii) the appearance of chromosomal aggregates resembling cytological figures observed during meiotic prophase I (Bowles et al., 2006). As a simultaneous exposure to ketoconazole and to the pan-RAR inverse agonist BMS204493 prevents these meiotic events, this suggests that ketoconazole, although not CYP26B1-specific, induces untimely meiosis in the testis through an inappropriate 
induction of the RAR signaling pathway (Koubova et al., 2006). The role of CYP26B1 in preventing meiosis entry in the fetal testis was confirmed in vivo by functional analysis of Cyp26b1-/ mutants: meiotic GC expressing Sycp3 are observed at ED13.5, at the time when GC in ovaries enter meiosis, in sharp contrast to the normal situation where meiosis initiates in the testis at the earliest by post-natal day (PND) 7 (MacLean, $\mathrm{Li}$, Metzger, Chambon, \& Petkovich, 2007). Altogether, these findings are compatible with the possibility that CYP26B1 stands for or synthesizes the MPS.

\subsection{CYP26B1 prevents proliferation as well as death of male gonocytes}

In the mouse testis Cyp26b1 is also expressed during the ED12.5-ED14.5 period, when male gonocytes undergo mitotic arrest in G0/G1 (quiescence) (Western, Miles, van den Bergen, Burton, \& Sinclair, 2008; Fig. 3). Pharmacological inhibition of CYP26 activity in organ culture of testes prevents the mitotic arrest of male GC and induces their death by apoptosis. These data suggest that impairing CYP26 action in the male fetal gonad, not only prevents gonocytes from entering meiosis, but also allows the establishment of their quiescent state and survival (Trautman et al., 2008). Similar conclusions were reached by Li, MacLean, Cameron, Clagett-Dame, \& Petkovich (2009) who generated a Sertoli cell-specific knockout of Cyp26b1 starting at ED15 (Cyp26b15C-/SC- mutants). In these mutants, some gonocytes exited from the G0 stage to re-enter the mitotic cycle, expressed SYCP3, indicating that they underwent inappropriate initiation of meiotic prophase I, and were subsequently lost from the seminiferous cords before birth. It is however noteworthy that ablation of Cyp26b1 in Sertoli cells most probably accounts only very partially for the functions of the enzyme in the developing testis, because the large majority of Cyp26b1 transcripts is detected outside the fetal seminiferous cords, in the somatic cells located in the interstitial spaces (Abu-Abed et al., 2002; Fig. 3) and is clearly confined to the peritubular myoid cells from birth (PNO) onwards (Vernet et al., 2006a; our unpublished results). It is therefore not surprising that another study showed that ablation of CYP26B1 activity in Sertoli cells from ED15 onwards has in fact little impact on adult spermatogenesis (Hogarth et al., 2015b). The combined results of these pharmacological and genetic experiments altogether suggest that increased ATRA levels induce aberrant entry of into meiosis, inappropriate cell proliferation and massive cell death amongst male gonocytes.

Thus, if exposure to ATRA is deleterious to male gonocytes, what might be the physiological significance of the strong and steady expression of RALDH1 in the immature Sertoli cells forming the cords of the fetal testis (Bowles et al., 2009; Fig. 3)? In an attempt to answer this question, we analyzed the outcome of ablating all RALDH genes in Sertoli cells, as early as ED15 (Aldh1a1-3Ser-/- mutants) (Raverdeau et al., 2012). At ED18.5, about 24 hours before birth, all gonocytes in testes of wild-type (WT) and Aldh1a1-3Ser-/- mutants are mitotically arrested (Fig. 4). They express the receptor tyrosine kinase KIT, a general marker of gonocytes (Fig. 4), but never GFRA1 nor ZBTB16, both of which are markers of undifferentiated spermatogonia. Then, WT and Aldh1a1-3Ser-/- mutant gonocytes express GFRA1 and ZBTB16 at PND1 (Fig. 4), resume proliferation by PND2 and migrate to the basement membrane until PND6 to adopt a spermatogonial phenotype (Kluin, Kramer, \& de Rooij, 1982; Nagano et al., 2000). As RALDH1 in Sertoli cells is the main source of ATRA for male fetal GC, this finding indicates that ATRA is not physiologically required to control the mitotic arrest of gonocytes, their migration from the center of the testicular cords to the basement membrane and their transition to spermatogonia. The reason for the robust expression 
of RALDH1 in Sertoli cells before birth is therefore still elusive. It is however noteworthy that RALDH1 exhibits a broad substrate specificity and can function as a detoxifying enzyme for xenobiotics (Alnouti \& Klaassen, 2008). Whether this is indeed the case during testis development remains to be tested.

\subsection{ATRA is required for the production of "differentiating" spermatogonia from male gonocytes}

In the mouse, the transition from gonocytes to spermatogonia occurs during the perinatal period, prior to the onset of prepuberty. Male gonocytes are the precursors of the stem spermatogonia and can also directly differentiate, without passing by the stem cell stage, into differentiating spermatogonia that support the first wave of spermatogenesis (Yoshida et al., 2006). Moreover, it has been suggested that gonocytes that fail to differentiate into spermatogonia may provide the source for formation of carcinoma in situ, the precursor lesion of most testicular germ cell cancer in humans (Soone et al., 2009). For these reasons, the transition from gonocytes to spermatogonia is an important step of the differentiation pathway leading to male gametes.

We found that GC from Aldh1a1-3Ser-/ mutant definitively loose KIT at PND3 as they do not reinitiate the transcription of the Kit gene, as it is normally the case at PND3 (Yoshida et al., 2006; Fig. 4). Moreover, germ cells from Aldh1a1-3Ser-/- never express STRA8 in contrast to WT gonocytes (Fig. 4). This latter observation is in keeping with the previous finding that expression of Stra8 is delayed in spermatogonia of neonatal Aldh1a1-/ mutants (Vernet et al., 2006b). The data presented here thus indicate that ATRA synthesis by Sertoli cells is necessary after birth for the production, from gonocytes, of the spermatogonia which initiate the first wave of spermatogenesis (Yoshida et al., 2006).

\subsection{ATRA possibly acts as a MIS for female gonocytes}

It is now admitted that ATRA acts as a MIS (Griswold, Hogarth, Bowles, \& Koopman, 2012). Nonetheless, the tissue from which originates the ATRA that induces meiosis in mouse fetal ovaries was disputed for several years. Studies of mice carrying an ATRA-dependent reporter transgene (RARE-hsp90-LacZ; Rossant, Zirngibl, Cado, Shago, \& Giguère, 1991), revealed that the responsiveness to ATRA is weak in the gonads, but robust in the mesonephroi of both sexes (Bowles et al., 2006; Koubova et al., 2006). As, both Aldh1a2 mRNA (Bowles et al., 2006) and RALDH2 protein (Fig. 3) are detected in mesonephroi of both sexes from ED11.5 to ED13.5, but not in the gonad itself, it was concluded that the source of ATRA detected in the ovary at the time of meiotic initiation is the adjacent mesonephros. Together these findings generated a model for the sex-specific timing of meiosis entry, according to which ATRA synthesized by the mesonephros serves as an extrinsic MIS acting in the adjacent gonad, unless degraded by CYP26B1, the MPS.

It is however noteworthy that the coelomic epithelium, which covers the embryonic gonad, also strongly expresses both Aldh1a2 transcripts (Niederreither, McCaffery, Dräger, Chambon, \& Dollé, 1997) and RALDH2 protein (Fig. 3). Thus, it can also function as a source of ATRA, closer to GC than the mesonephros. On the other hand, Kumar et al. (2011) have challenged the view that ATRA is required for entry of ovarian gonocytes in meiosis. Actually, by analyzing mice carrying null mutations for RALDH2, singly or in combination with null mutations for RALDH3 


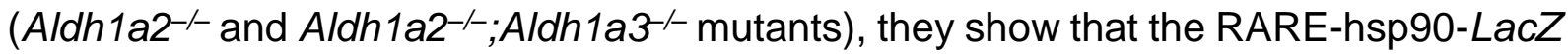
activity typically observed throughout the normal mesonephros and the gonads is totally eliminated, as expected. Yet, both mutant and WT ovaries exhibit similar expressions of meiotic effectors such as Stra8, Sycp3 and phosphorylated H2AX histone, which marks DNA double-strand breaks occurring during meiotic recombination. The authors conclude that meiosis occurs normally, the lack of detectable ATRA in the vicinity of the ovaries. Nevertheless, these observations left open the possibility that RALDH1 might be also involved. This hypothesis has been neglected for a long time likely because (i) Aldh1a1-/ mice are viable and fertile (Fan et al., 2003; Matt et al., 2005); (ii) RALDH1 has the lowest affinity for RAL, being about 15-fold less efficient than RALDH2 at producing ATRA (Gagnon, Duester, \& Bhat, 2002); and (iii) RALDH1 has been proposed to be marginally involved in ATRA synthesis in vivo (Niederreither, Vermot, Fraulob, Chambon, \& Dollé, 2002). The fact remains that both Aldh1a1 mRNA and RALDH1 protein are detected in somatic cells of the developing gonads which are in direct contact with gonocytes (Fig. 3), and meiosis is indeed delayed in the Aldh1a1-/ ovary, as judged by the expression of marker genes (Bowles et al., 2016). Thus, in spite of its low capability of ATRA synthesis, RALDH1 activity appears to participate in meiotic initiation in female GC. By

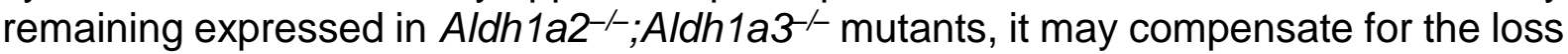
of the two other ATRA-synthesizing enzymes. The observation that cultured GC upregulate meiotic genes in response to $1 \mathrm{nM}$ ATRA supports the view that ATRA at low concentrations is able to favor meiosis (Bowles et al., 2010; Tedesco, Desimio, Klinger, De Felici, \& Farini, 2013).

\subsection{Stra8 as the meiotic gatekeeper}

A wealth of data support the model whereby ATRA acts as the MIS by inducing the expression of STRA8, a $45 \mathrm{kD}$ vertebrate-specific protein (Oulad-Abdelghani et al., 1996). STRA8 actually functions as a "meiotic gatekeeper" by controlling the switch between GC division by mitosis and meiosis, as evidenced by gene knockout studies in the mouse (Baltus et al., 2006; Anderson et al., 2008; Mark et al., 2008). The Stra8 promoter contains a RARE which can bind to RAR/RXR heterodimers, making this gene a possible direct target of ATRA (Kumar et al., 2011; Raverdeau et al., 2012). Moreover, the normal expression of Stra8 in female gonocytes is inhibited in fetal ovaries cultured in the presence of the pan-RAR inverse agonist BMS-204493 (Koubova et al., 2006), and ATRA at supra-physiological levels can stimulate expression of Stra8 when added to the medium of cultured embryonic testes from mice (Baltus et al., 2006; Bowles et al., 2006; Koubova et al., 2006), humans (Le Bouffant et al., 2010) and amphibians (Wallacides, Chesnel, Chardard, Flament, \& Dumond, 2009; Piprek et al., 2013), suggesting that the requirement of ATRA for meiosis entry could be a conserved mechanism in tetrapods.

These data must, however, be interpreted with caution for three reasons. First, it has been recurrently emphasized that developmental alterations generated by added ATRA do not necessarily reflect cellular processes operating in real life (Horton \& Maden, 1995; Mark, Ghyselinck, \& Chambon, 2006). Second, neither expression Stra8 nor up-regulation of meiotic markers is synonymous of entry into meiosis. For instance, expression of STRA8 and proteins associated with key meiotic processes occurs in spermatogonia, well before the appearance of premeiotic spermatocytes (Mark, Teletin, Vernet, \& Ghyselinck, 2015; Evans, Hogarth, Mitchell, \& Griswold, 2014). Third, silencing of Stra8 expression by the pan-RAR inverse agonist BMS-204493 
does not necessarily reflects impairment of its induction by endogenous ATRAactivated RAR because this synthetic retinoid stabilizes interaction between corepressor complexes and RAR, whether naturally bound by ATRA or not. By tethering corepressor-associated histone deacetylase activities, it may silence genes containing RARE but not necessarily activated by endogenous ATRA-bound RAR (Germain et al., 2009). Thus, although STRA8 is indisputably instrumental to proper meiosis, the control of its expression by endogenous ATRA in female GC is more controversial.

\subsection{An alternative viewpoint of meiosis induction in female gonocytes}

Interpretations of the data showing an involvement of ATRA in meiosis are guided by the prevailing idea that both a MPS and a MIS are required to explain the sexspecific timing of meiotic initiation (reviewed in Bowles \& Koopman, 2007; Kocer, Reichmann, Best, \& Adams, 2009; Griswold, Hogarth, Bowles, \& Koopman, 2012). This is mainly due to the pioneering finding that Cyp26b1, whose function is supposedly to degrade ATRA, is expressed in the male, but not in the female fetal gonad where meiosis initiates. Since then, Kumar et al. (2011) have proposed that CYP26B1 may instead metabolize a substrate that is distinct from ATRA (for instance another aldehyde). It must be emphasized that considering the "MPS only" hypothesis to interpret the current data would reconcile the apparent discrepancies existing in the literature. According to this scenario, inducing quiescence instead of meiosis in male gonocytes would require a yet unknown substance produced by CYP26B1 (the MPS). On the opposite, entering meiosis in female gonocytes would be an autonomous, intrinsic, property of GC without the requirement of any MIS, including ATRA synthesized by RALDH1 or another RALDH. Interestingly, several authors propose that ATRA in the fetal ovary is involved in GC proliferation rather than meiosis initiation (Koshimizu, Watanabe, \& Nakatsuji, 1995; Morita \& Tilly, 1999; Trautmann et al., 2008; Jørgensen et al., 2015).

\section{ATRA SIGNALING IS INSTRUMENTAL TO SPERMATOGONIA DIFFERENTIATION IN THE PREPUBERTAL AND ADULT TESTIS}

Spermatogenesis is initiated during the prepubertal period in the seminiferous epithelium (SE) and then proceeds throughout adulthood. In the mouse, the initial wave of spermatogenesis, which starts around PN2, and is completed by PN35, involves a subpopulation of gonocytes (Yoshida et al., 2006). The subsequent prepubertal and all adult waves derive only from spermatogonia. These cells have traditionally been classified into "undifferentiated" and "differentiating" based on their ability to self-renew and differentiate (de Rooij \& Russell, 2000). Undifferentiated type A (Aundiff) spermatogonia are endowed with stem cell functions: they can (i) maintain their own population, thereby ensuring long term maintenance of the germline and (ii) produce daughter cells that differentiate into spermatozoa. Based on whole-mount analyses of seminiferous tubules, Aundiff spermatogonia collectively correspond to $A_{\text {single }}\left(A_{s}\right.$, isolated single) spermatogonia which, upon division, give rise to syncytia consisting of $2 A_{\text {paired }}\left(A_{p r}\right), 4 A_{a l i g n e d-4}\left(A_{a l-4}\right), 8\left(A_{a l-8}\right), 16$ (Aal-16) cells and occasionally 32 cells interconnected by cytoplasmic bridges resulting from incomplete cytokinesis (reviewed in de Rooij \& Russell, 2000; Fig. 5). Cell lineage tracing experiments indicate that a subpopulation of $A_{s}$ spermatogonia is responsible for the stem cell function in steady state spermatogenesis but its $A_{a l}$ progeny retains the potential to revert to $A s$ and 
reconstitute the entire spermatogenesis when the tissue is damaged or when these cells are transplanted into GC-depleted seminiferous tubules (Hara et al., 2014; and references therein; Fig.5). Aundiff spermatogonia are evenly distributed along seminiferous tubules. They periodically exit from the stem cell pool and differentiate without a mitotic division into differentiating, type $A_{1}$ spermatogonia. This step known as the $A_{\text {aligned }}$ to $A_{1}\left(A_{a l}-A_{1}\right)$ transition is considered as the entrance in the differentiation pathway ultimately yielding spermatozoa. Five mitotic divisions follow $A_{1}$ formation, producing sequentially $A_{2}, A_{3}, A_{4}$, intermediate (Int) and $B$ spermatogonia and accompanying elongation of the syncytial chains. Types $A_{1}$ to $B$ spermatogonia referred to as "differentiating spermatogonia" are committed towards the pathway yielding spermatocytes (Fig. 5). They lose their capacity for self-renewal (Shinohara, Orwig, Avarbock, \& Brinster, 2000) and differentiate at precise intervals along the seminiferous tubules, each step of differentiation being coupled with a mitotic division (de Rooij, 1998).

KIT and STRA8 are restricted to differentiating spermatogonia and the onset of their synthesis, detected by IHC (and/or by ISH) during the progression of spermatogonia differentiation, is a commonly accepted hallmark of the $\mathrm{A}_{\mathrm{al}} \mathrm{A}$ - 1 transition (Schrans-Stassen, van de Kant, de Rooij, \& van Pelt, 1999; Ghyselinck et al., 2006). Other proteins that are linked with cell fate or function represent useful tools to characterize the state of spermatogonia differentiation in immunohistochemical assays (Fig. 5).

\subsection{The transition from undifferentiated to differentiating spermatogonia critically relies on ATRA}

The finding that ATRA is a key regulator of the $A_{a l}-A_{1}$ transition was brought about by vitA deficiency studies. Depriving adult rodents in vitA induces a rapid loss of meiotic spermatocytes and spermatids, ultimately yielding seminiferous tubules containing only Sertoli cells, spermatogonia and, in the case of rats, preleptotene spermatocytes. Based on cell kinetic properties and on the absence of Kit expression, the remaining spermatogonia in vitA-deficient animals are arrested just before their differentiation into $A_{1}$ spermatogonia. In rats and mice, reintroduction of vitA in the diet or systemic administration of different retinoids induces, within 24 hours, the $A_{a l}-A_{1}$ transition and, subsequently, the reinitiation of spermatogenesis in most of the tubules (van Pelt et al., 1995; Gaemers, van Pelt, van der Saag, \& de Rooij, 1996; Schrans-Stassen, van de Kant, de Rooij, \& van Pelt, 1999; and references therein).

The presence of seminiferous tubule segments in which spermatogenesis is not restored several months after vitA replacement in vitA-deficient animals raises the question as to whether retinoid are also required for $A_{\text {undiff }}$ Spermatogonia self-renewal or survival, in addition to be instrumental to the $A_{a}$ al- $A 1$ transition. A recent work using pulse labelling of spermatogonia subtypes in a vitA-deficient mouse model demonstrates that the GFRA1-positive spermatogonia, which comprises the actual stem cell pool, does not depend on ATRA signaling for self-renewal. ATRA is neither required for their differentiation into NGN3-positive Aundiff spermatogonia. However, NGN3-positive spermatogonia, a poised cell population that differentiates further into KIT-positive spermatogonia in homeostatic spermatogenesis, cannot do so and die in the absence of vitA (Ikami et al., 2015). Altogether, these data indicate that ATRA is indispensable in vivo to trigger the $A_{a l}-A_{1}$ transition and is independently required for the survival of some Aundiff Spermatogonia. In contrast, stem spermatogonia can survive 
and divide without ATRA. Whether ATRA is also required in differentiating spermatogonia has not been investigated so far.

\subsection{The sources of ATRA destined for spermatogenesis are intrinsic to the SE}

A major source of ATRA in the SE was identified using the Aldh1a1-3 ${ }^{\text {Ser-/- }}$ mutant mouse line as a model. In steady-state conditions, the most advanced GC ever present in Aldh1a1-3Ser-/- mutants are ZBTB16 positive, but STRA8 and KIT negative, Aundiff spermatogonia (Raverdeau et al., 2012). Interestingly, almost all these cells strongly express RARG indicating that they have the capacity to respond to ATRA. However, spermatogonia cannot cell-autonomously trigger the $A_{a l}-A_{1}$ transition because they do not possess the enzymatic machinery to make ATRA (Evans, Hogarth, Mitchell, \& Griswold, 2014). Within twenty-four hours following an injection of ATRA, a majority of spermatogonia in Aldh1a1-3 ${ }^{\text {Ser-/- }}$ seminiferous tubules switch to a STRA8- and KITpositive phenotype. It is thus concluded that the only source of ATRA in the SE at the onset of the prepubertal period is RALDH activity in Sertoli cells and that it acts in a paracrine manner to instruct the initial $A_{a l}-A_{1}$ transition.

Quite strikingly, spermatogenesis continues for months in Aldh1a1-3 ${ }^{\text {Ser-/- }}$ mutants after rescue by an injection of ATRA. This situation cannot be accounted for by a persistent effect of exogenous ATRA because its half-life is less than 1 hour in the mouse and direct measurements of ATRA levels in testes indicate that its residual amount 4 days following its injection is null (Hogarth et al., 2015a). Thus, once the first wave of spermatogenesis has progressed beyond a certain point, RALDH activity in Sertoli cells is no longer required for spermatogonia differentiation. (Raverdeau et al., 2012). In this context, ATRA is necessarily produced either outside or within the SE but not in Sertoli cells. The interstitial spaces between seminiferous tubules contains two potential sources of ATRA for the SE: Leydig cells, which strongly and steadily express RALDH1, and capillaries that covey ATRA derived from the blood (Kane, Folias, Wang, \& Napoli, 2008; Vernet et al., 2006a). ATRA from the interstitial tissue is however unable to trigger spermatogenesis in Aldh1a1-3 Ser-/- mutants. This is likely due to the activity of peritubular myoid cells that surround the seminiferous tubules. Actually from birth to adulthood, these cells strongly express the CYP26A1 and CYP26B1 enzymes, whereas CYP26 are undetectable by ISH and IHC in the other cell populations of the post-natal testis (Vernet et al., 2006a; Wu, Wang, Guo, \& Xu, 2008). Interestingly, administration of the CYP26 inhibitor liarozole to Aldh1a1-35er-/mutants can restore a complete spermatogenesis (Fig. 6). This experiment indicates that the catabolic barrier formed by the peritubular myoid cells is efficient in Aldh1a1$3^{\text {Ser-/- }}$ mutants and probably also functions in WT mice to degrade any ATRA present in the immediate environment of the seminiferous tubules, thus preventing inopportune occurrences of $A_{a l}-A_{1}$ transitions. Since ATRA present around the tubules is unable to reach spermatogonia in Aldh1a1-3 ${ }^{S e r-/-}$ mutants, the activity required to sustain their differentiation is necessarily supported by their progeny: RALDH2 in spermatocytes and spermatids is clearly the only possible source of ATRA for spermatogenesis in these mutants (Vernet et al., 2006a; Sugimoto, Nabeshima, \& Yoshida, 2012), where it acts in a paracrine fashion to induce spermatogonia differentiation from the second round of spermatogonia differentiation onward.

Ablation of $R d h 10$ in the SE also causes a block in spermatogenesis at the $A_{a l}-$ $\mathrm{A}_{1}$ transition, which is rescued when RAL or ATRA are administered exogenously.

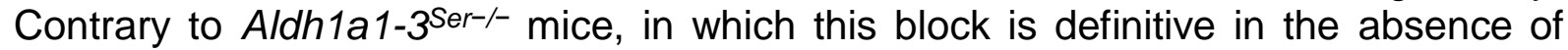
retinoid treatment, spermatogenesis is spontaneously restored to normal in young in 
the Rdh10-deficient adults at PNW9, suggesting that the first step in the synthesis of ATRA (transformation of vitA into RAL) is performed by an enzyme other than RDH10 in adult testis (Tong, Yang, Davis, \& Griswold, 2013). Interestingly, results similar to what is observed in Aldh1a1-3 Ser-/- and Rdh10-deficient mutants are also seen following pharmacological inhibition of RALDH: when prepubertal mice were treated with WIN 18,446, the only GC type present within their seminiferous tubules is Aundiff spermatogonia (Hogarth et al., 2013).

\section{ATRA IS INSTRUMENTAL TO MEIOSIS IN SPERMATOCYTES}

It is commonly believed that since expression of Stra8 in GC is indispensable for their entry into meiosis, then expression of Stra8 also indicates their irreversible commitment towards meiosis (references in Mark, Teletin, Vernet, \& Ghyselinck, 2015). This may eventually apply to the situation in ovary where expression Stra8 by fetal gonocytes is temporally tightly correlated with the initiation of meiosis, as it precedes by only a few hours the expression of markers of meiotic prophase I. In contrast, in the developing and adult testis Stra8 expression in spermatogonia precedes their meiotic entry by about a week (Drumond, Meistrich, \& Chiarini-Garcia, 2011; Kluin, Kramer, \& de Rooij, 1982; Mark, Teletin, Vernet, \& Ghyselinck, 2015; Zhou et al., 2008). Additionally, synthesis of Stra8 mRNA and protein is biphasic in spermatogonia: both are detected in differentiating type A spermatogonia, but not in intermediate nor in type B spermatogonia, the latter representing the immediate precursors of preleptotene spermatocytes. Spermatocytes in S (preleptotene) phase reinitiate Stra8 expression before entering meiosis (Vernet et al., 2006b; Mark, Teletin, Vernet, \& Ghyselinck, 2015; Fig. 5).

In the male mouse, meiotic initiation occurs for the first time during prepuberty at about PND7, then meiosis recurs repeatedly and continuously throughout adult life (Drumond, Meistrich, \& Chiarini-Garcia, 2011; Kluin, Kramer, \& de Rooij, 1982). The involvement of ATRA in this process was investigated using Aldh1a1-3 ${ }^{\text {Ser-- }}$ mutants in which spermatogenesis was rescued by an injection of ATRA to resume the $A_{a l}-A_{1}$ transition (Raverdeau et al., 2012). Under these conditions, preleptotene spermatocytes expressing Stra8 and $R e c 8$ (encoding a meiosis specific recombinase) appear, synchronously, 8 days post-ATRA injection in all seminiferous tubules. Treatment with the pan-RAR inverse agonist BMS-204493 prior to and during meiotic initiation in rescued Aldh1a1-3Ser-/- mutants abrogates Stra8 expression and induces cytological abnormalities which are identical to those displayed by Stra8-null spermatocytes. Actually, in the absence of STRA8 expression, leptotene spermatocytes undergo premature chromosome condensation yielding hyper condensed chromatin figures consisting in 40 univalent chromosomes which are thus similar to mitotic metaphases (Mark et al., 2008). The potential source of ATRA in Aldh1a1-3 ${ }^{\text {Ser- }-}$ mutants at the time of meiotic initiation is represented by spermatocytes, which express RALDH2 (Vernet et al., 2006a; Raverdeau et al., 2012). Keeping in mind the possible artefactual effects of BMS-204493 mentioned above, these data may indicate a role for endogenous ATRA in the stable commitment to the meiotic cell cycle in the testis. A recent report further suggests that ATRA may also be required for meiotic recombination (Kent et al., 2016). 


\section{ATRA METABOLISM WITHIN THE SE CONTROLS THE TIMING AND SPATIAL PATTERNING OF SPERMATOGONIA DIFFERENTIATION}

At a given point along a murine seminiferous tubule, the transition from $A_{\text {undiff }}$ to differentiating spermatogonia proceeds in a periodic manner, every 8.6 days, while their descendants are generated and displaced at a constant pace towards the lumen of the seminiferous tubules. These processes result in the formation of stratified, recurring, cellular associations of fixed composition between precise subtypes of spermatogonia, spermatocytes and spermatids, called stages of the SE cycle, each epithelial stage spanning a short segment along a tubule. Twelve stages (I to XII), are recognizable in the mouse SE, with stage I corresponding to the first step of spermatid maturation (reviewed in Russell, Ettlin, \& Clegg, 1990; de Rooij, 1998; Fig. 7). The Aal$A_{1}$ transition is the point of entry into the SE cycle, and four turns of this cycle are required for a $G C$ to progress from a type $A_{1}$ spermatogonium to a spermatozoon that is ready to be released in the tubule lumen. The SE cycle, as well as the repetitive patterning of GC associations along the seminiferous tubules, called wave of the SE, represent the keys for the physiologically asynchronous GC differentiation that allows a constant production of mature spermatids by the testis and a continuous release of spermatozoa in the epididymis. It is therefore paramount to the successful maintenance of fertility throughout the reproductive lifetime of males.

\subsection{The SE cycle and wave both rely on retinoid signaling}

The idea that retinoids could play a role in controlling the SE cycle came from observations made on vitA-deficient rats in which spermatogenesis was restored upon systemic administration of vitA and/or ATRA. Actually in retinoid-rescued rat testes, the full representation of the normal 14 stages of the SE is no longer seen. Instead, they contain only 3-4 stages, which are present at abnormally high frequencies, yielding an apparent "truncation" of the wave. Accordingly, the spermatogenesis of these animals is described as "synchronous", referring to the near-identical timing of GC progression in the entire testis (Morales \& Griswold, 1987; vanPelt \& de Rooij, 1990; Van Beek \& Meistrich, 1990). More recently, it was shown that exposure of vitAsufficient prepubertal mouse testis to exogenous ATRA drives the entire Aundiff population to enter the differentiation pathway in the whole testis, resulting also in synchronous spermatogenesis at adulthood (Snyder, Davis, Zhou, Evanoff, \& Griswold, 2011; Davis, Snyder, Hogarth, Small, \& Griswold, 2013).

VitA is also indispensable for the maintenance of the epithelial stages. This role was inferred from the study of $R b p 4^{-/}$mutant mice which are unable to mobilize their ROL liver stores because they lack the blood carrier Retinol-Binding Protein (Ghyselinck et al. 2006). In these mutants, the vitA-deficiency-induced defects progress over a period of several weeks, much earlier than in WT mice. Therefore, their timeline can be drawn, which allows investigating the target cells and physiological functions of retinoids in the SE. The seminiferous tubules of vitA-deficient $R b p 4^{-/}$mice display normal segments at any of the 12 stages of the cycle that co-exist with segments lacking one or two GC layers (e.g., entire spermatocyte or spermatid layers; Fig. 7). Importantly, these abnormal segments can all be classified as variants of the stages of the SE cycle, and aberrant cellular associations that would not fit in one of the canonical 12 stages are never found. Therefore, the tight coordination between spermatogonia differentiation and steps of meiosis and spermiogenesis is 
maintained in conditions of decreased vitA availability. These observations lead to the conclusion that a major mechanism accounting for vitA-deficiency-induced failure of the SE cycle is the normal maturation of spermatocytes and spermatids in a situation where spermatogonia differentiation is temporarily arrested (Ghyselinck et al., 2006).

\subsection{Endogenous ATRA levels in the SE are tightly regulated}

Several observations support the notion that endogenous ATRA also regulates the SE cycle and wave. First, expression of the ATRA-target genes Stra8 in spermatocytes, and Stra6 in Sertoli cells peak at stages VII-VIII of the SE cycle (Vernet et al., 2006b). Second, ATRA is crucial for cellular processes which occur at stages VII-VIII of the cycle including $A_{a l}-A_{1}$ transition, entry of spermatocytes into meiosis and release of mature spermatids (spermiation) into the lumen of the seminiferous tubules (Raverdeau et al., 2012; Fig. 7). Third, measurement of ATRA levels in synchronized mouse testes support the view that an ATRA pulse occurs at stages VII-IX of the SE cycle (Hogarth et al., 2015a; Fig. 7). Altogether, these observations suggest that ATRA is only along distinct segments of the seminiferous tubules.

The bioavailability of ATRA is controlled by regulation of the balance between its production and degradation. No variations in the levels of CYP26 enzymes exist across the SE cycle (Vernet et al., 2006a; Hogarth et al., 2015b). In contrast, several proteins involved in retinol storage and production of RAL represent potential candidates for contributing to how ATRA levels are regulated in a periodic manner along seminiferous tubules. The distribution pattern of Lrat transcripts in spermatids supports the possibility that retinoid storage is promoted during stages I-VI of the SE cycle (Vernet et al., 2006a). Rdh10 and dehydrogenase/reductase (SDR family) member 4 (Dhrs4) display SE stage-specific expressions, suggesting that conversion of vitA to RAL may occur only at specific sites along seminiferous tubules. However, the fact that $98 \%$ of the mutant mice lacking $R d h 10$ display a spermatogenesis with a normal frequency of the epithelial stages, indicates that RDH10 activity is not indispensable to regulate the wave of the SE cycle (Tong, Yang, Davis, \& Griswold, 2013).

\subsection{RALDH activity regulates the spermatogenic wave}

The expression patterns of Aldh1a1 to Aldha3 genes has been extensively investigated: RALDH1 and RALDH2 are the main ATRA synthesizing enzymes in Sertoli cells and GC respectively, while RALDH3 is not detected in the SE (Vernet et al., 2006a, Bowles et al., 2009; Sugimoto, Nabeshima, \& Yoshida, 2012; Kent et al., 2016). In Sertoli cells of the adult testis, Aldh1a1 transcripts are expressed in a SE cycle-dependent manner with the highest levels being found at stages II-VII (Fig. 8). In GC, Aldh1a2 transcripts are expressed in a periodic manner with a peak in late pachytene and diplotene spermatocytes, at stages VIII to XII (Vernet et al., 2006a; Fig. 8). These periodic expressions likely reflect a functional role of RALDH. Interestingly, histological analyses show that the spatial distribution of the different SE stages is markedly altered in the testes of Aldh $1 a 1^{-1-}$ males: cross sections of the SE at the same stage are often assembled in defined territories that, according to anatomy, corresponds to a given seminiferous tubule (Nakata et al., 2015). This similarities strongly suggest that, the segment length occupied by a given stage is increased in Aldh1a1-- mice. In contrast, the topography of the SE stages does not display any defined profile in WT testes (Fig. 8). This points to a hitherto ignored function of $\mathrm{RALDH} 1$ in regulating the wave of the SE cycle. 
RALDH2 in spermatocytes and spermatids is sufficient to maintain a SE cycle in the complete absence of RALDH in Sertoli cells (Raverdeau et al., 2012). This observation suggests a role of ATRA synthesized by GC in regulating the SE cycle or wave. We have proposed that, in adult males, late pachytene spermatocytes present at stage VIII may control the tight coordination between the differentiation of $A_{a l}$ spermatogonia and entry into meiosis through cyclically modulating the ATRA environment of the cells (Raverdeau et al., 2012). To further establish the specific role of RALDH2 in the testicular ATRA biosynthesis, a GC specific ablation of Aldh1a2 is needed to circumvent the problem of embryonic lethality of the Aldh1a2 knockout mice.

\section{MALE GC ARE BOTH DIRECT AND REMOTE TARGETS OF ATRA ACTION: LESSONS FROM MOUSE MUTANTS LACKING RETINOID RECEPTORS}

\subsection{RAR in fetal GC differentiation}

All 3 RAR isotypes are reported to be expressed in fetal testes and ovaries (Bowles et al., 2006). In mice lacking one RAR isotype, testis differentiation proceeds normally up to the start of puberty ( Rara $^{-/}$and Rarg $^{-/}$mutants) or even beyond its completion (Rarb- ${ }^{-1}$ mutants) in males, while females display a normal ovarian morphology and are fertile at adulthood. Compound mutants lacking 2 RAR isotypes die in utero or at birth and display multiple defects in many tissues, the spectrum of which varies between individuals (Mark, Ghyselinck, \& Chambon, 2006). Their gonadal sex can be unambiguously diagnosed based on the arrangement of the somatic cells into compact cords, in male fetuses, as opposed to epithelial clusters with ill-defined borders, in female fetuses. Histological criteria are however far from being sufficient to assess the normality of the developing gonads. This is especially true for young Rar compound knockout fetuses, where variations in the timing of GC differentiation, already patent in the WT situation, seem to be exacerbated. It is nevertheless interesting to note that while reanalyzing the serial histological sections obtained more than 20 years ago from the three ED18.5 Rara $^{-\alpha} ;$ Rarg $^{-1}$ female fetuses ever produced so far (Mendelsohn et al., 1994), we discovered in preparing this review that one fetus completely lacked meiotic oocytes (Fig. 9). Demonstrating that this finding actually reflects GC autonomous effects of the absence of RAR awaits the generation of a genetic model in which signaling via all 3 RAR is abrogated specifically in GC, as a mean of eliminating any functional redundancy.

\subsection{RARG controls the capacity of spermatogonia to respond to ATRA}

Aundiff spermatogonia are present throughout the cycle of the SE, including stage VIII when the peak of ATRA concentration supposedly induces the $A_{a l}-A_{1}$ transition (Hogarth et al., 2015a; de Rooij \& Russell, 2000). Therefore, a mechanism must exist that ensures the preservation of a stem cell pool while producing, at the same time, differentiating cells upon exposure to ATRA. Recent observations suggests that this mechanism relies on the differential expression of Rarg between subpopulations of undifferentiated spermatogonia: as opposed to their $A_{a l}$ progeny, $A_{s}$ spermatogonia do not express Rarg and therefore maintain an undifferentiated state and self-renewal capabilities even in the of presence of high ATRA levels in the SE (Gely-Pernot et al., 2012; Ikami et al., 2015). 
The role of RARG in spermatogonia differentiation was initially inferred from the phenotypic analysis of Rarg knockout mice (Rarg ${ }^{-1}$ mutants). The seminiferous tubules of sexually mature, young (2-3 month-old), Rarg $^{-1}$ mutants display segments lacking one or two GC layers around their entire circumference, thereby forming variants of the stages of the SE cycle, identical to those observed in vitA-deficient Rbp4-/ mice (Ghyselinck et al., 2006; Gely-Pernot et al., 2012). Since Rarg-/spermatocytes and spermatids do not display features reminiscent of increased apoptosis or delayed differentiation, the presence of these variants most likely reflects the fact that the $A_{a l}-A_{1}$ transition is temporarily arrested. In some cases the $A_{a l}-A_{1}$ transition appears to take place in due time, yielding normal stages, while in other instances no $A_{1}$ spermatogonia are formed and $A_{a l}$ spermatogonia have to wait one (or several) $S E$ cycles to transition to $A_{1}$, yielding seminiferous tubule segments with missing generations of spermatocytes and spermatids. Because these variants also occur upon ablation of Rarg in spermatogonia only (RargSpg-/ mutants), it is concluded that RARG functions cell-autonomously in spermatogonia to control the $A_{a l}-A_{1}$ transition.

With respect to progression with ageing of testis defects, Rarg $^{-/}$mutants are similar, but not fully identical, to vitA-deficient mice. Actually, seminiferous tubules of 1-year-old Rarg $^{-1}$ mutants contain only Sertoli cells and spermatogonia, some of which still express markers of differentiation (e.g., KIT). Thus, in contrast to the vitA-deficient situation, inactivation of Rarg is not sufficient to arrest all spermatogonia at the $A_{a l}-A_{1}$ transition. This discrepancy between the two situations is most readily accounted for by a functional redundancy between RARG and another RAR in spermatogonia, notably RARA which appears to be ubiquitously expressed at low levels not necessarily detected by IHC and ISH (Gely-Pernot et al., 2012). To get rid of any possible functional compensation, mice lacking all RAR in spermatogonia were generated

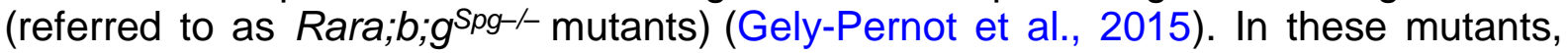
ablation of Rar genes is efficient, as early as PND5, i.e., shortly after the onset of spermatogenesis during prepuberty. In young adults, the phenotypic outcome of ablating all RAR in spermatogonia is undistinguishable from that induced upon Rarg knockout, indicating that RARG is indeed the major functional RAR isotype in spermatogonia. In addition, the SE of 1 year-old Rara;b;gSpg-/ mutants contains only KIT-negative Aundiff spermatogonia (in addition to Sertoli cells), demonstrating that RARA or RARB in spermatogonia can compensate, at least in part, for the loss of RARG.

\subsection{The response of spermatogonia to ATRA relies on RXR/RAR heterodimers}

The ATRA signal is generally transduced by RXR/RAR heterodimers, notably during mouse embryonic development (Mark, Ghyselinck, \& Chambon, 2006). However, it can also be relayed by RAR independently of RXR, as it is the case in Sertoli cells (Vernet et al., 2006b). To discriminate between these two possibilities in GC, mice lacking all RXR isotypes in spermatogonia, and hence also in their spermatocytes and spermatids descendants were generated (hereafter called $R \times r a ; b ; g^{S p g-/}$ mutants). Age-matched Rxra;b; $g^{\text {Spg-/ }}$ and Rara;b;gSpg-/ mutants display similar, if not identical, phenotypes indicating that RXR and RAR exert convergent functions in spermatogonia. As in addition, both anti-RXR and anti-RAR antibodies precipitate, with similar efficiencies, DNA sequences containing the RAR binding sites of the Stra8 promoter, it is concluded that RXR/RAR heterodimers are the functional units transducing the ATRA-signal in spermatogonia (Gely-Pernot et al., 2015). 
To identify the ATRA-controlled genes in spermatogonia, organ cultures of Aldh1a1-3 ${ }^{\text {Ser-l- }}$ testes were treated with a RARG-selective agonist (BMS-189961) to initiate spermatogenesis. Microarray expression profiling identified Sall4, which encodes a zinc-finger transcription factor, amongst the few transcripts that are differentially expressed upon activation of RARG. Interestingly, mice deficient for Sall4 in spermatogonia display testis defects that resemble those observed in $R x r a ; b ; g^{S p g-/-}$ and Rara; $b ; g^{S p g-/}$ mutants, namely lack of differentiating spermatogonia (Hobbs et al., 2012). A RAR-binding region located in the first intron of Sall4 was identified. This region contains a RARE that binds to RXRA in combination with RARG, but not to RXRA nor to RARG alone, as revealed by electrophoretic mobility shift assays (GelyPernot et al., 2015). It is thus proposed that ATRA-activated RXRA/RARG heterodimers increase the level of SALL4A protein in spermatogonia, thereby allowing the increase in Kit expression (Fig. 10) that is a hallmark of the $A_{a l}-A_{1}$ transition (Schrans-Stassen, van de Kant, de Rooij, \& van Pelt, 1999). In agreement with this proposal, the amount of SALL4 protein detected in spermatogonia is higher at the $A_{1}$ stage than at the Aal stage (Hobbs et al., 2012). In addition to SALL4, a recent study shows that ATRA controls spermatogonia differentiation through activating replicationdependent core histone genes (Chen et al., 2016).

\subsection{RARA in Sertoli cells also contribute to ATRA functions in the SE}

It is intriguing that spermatogonia differentiation is not stopped straight away in Rara; $b ; g^{S p g-/}$ and $R \times r a ; b ; g^{\text {Spg-/- }}$ mutants, but instead proceeds during several weeks in young adults (Gely-Pernot et al., 2015). This observation suggests the existence of a subpopulation of $A_{a l}$ spermatogonia that are committed to become $A_{1}$ even though they are unable to cell-autonomously respond to ATRA. Given the pivotal role of ATRA in spermatogonia differentiation, instructing the $A_{a l}-A_{1}$ transition in this subpopulation may nevertheless require an ATRA signal mediated by the Sertoli cell, which expresses RARA and is the only somatic cell-type present in the SE. We propose that Sertoli cell responds to ATRA by synthesizing and secreting yet unknown paracrine signal(s) that stimulate a subpopulation of $A_{a l}$ spermatogonia to transition to $A_{1}$ with no need for functional RXR/RAR heterodimers within these spermatogonia. This proposal is supported by the observation that the only $\mathrm{GC}$ ever present in the SE of mice lacking

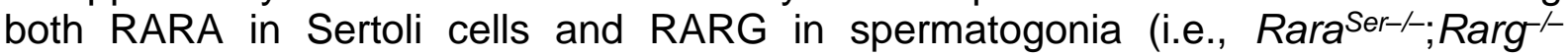
mutants) are undifferentiated spermatogonia, as it is the case when ATRA synthesis abolished in the SE (Gely-Pernot et al., 2015).

It is also interesting to note that, in addition to differentiating spermatogonia, spermatocytes are also generated in testes of Rara; $b ; g^{S p g-/}$ and $R \times r a ; b ; g^{S p g-/-}$ mutants. Moreover, meiosis proceeds at a normal pace, despite the absence of either RAR or RXR in all spermatocytes. Therefore, if ATRA is actually a mandatory MIS, then it operates in Sertoli cells rather than in GC (Gely-Pernot et al., 2015).

\section{CONCLUDING REMARKS}

An active ATRA signaling pathway is instrumental to the onset of oogenesis as well as to the initiation and maintenance of spermatogenesis in the mouse. Interestingly, it is also critically required in this species to disengage spermatozoa from the Sertoli cell cytoplasm during spermiation (Mark, Teletin, Vernet, \& Ghyselinck, 2015; and references therein; Fig. 7) and to promote the expulsion of the secondary oocyte from the mature follicle at ovulation (Kawai, Yanaka, Richards, \& Shimada, 2016; Fig. 1). Gametogenesis is similar in mice and humans, notably with respect to 
the subtypes of GC, the sequence of cellular events involved in the differentiation cascade and the expression of regulatory molecules (Wu et al., 2009). In humans, a direct effect of ATRA on meiosis has been shown only in the ovary (Le Bouffant et al., 2010). Nonetheless, the demonstration that the bis-(dichloroacetyl)-diamine WIN 18,446 exerts its deleterious effects on human sperm counts and SE histology through its property to inhibit RALDH activity supports the view that ATRA is also required for human spermatogenesis (Hogarth et al., 2013).

In this context it is worth noting that disturbances of the vitA metabolism caused by exposure to omnipresent environmental pollutants such as dioxin-like compounds or phthalates are strongly suspected to participate in the decrease of male fertility observed in industrialized countries (Novák, Benísek, \& Hilscherová, 2008; Sarath Josh et al., 2014). Moreover, possible side effects on human fertility of pharmacological doses of retinoids used in the treatment of acne have not been evaluated so far. In this context, it is crucial to decipher the molecular mechanisms by which ATRA controls gametogenesis.

\section{REFERENCES}

Abu-Abed, S., MacLean, G., Fraulob, V., Chambon, P., Petkovich, M., \& Dollé, P. (2002). Differential expression of the retinoic acid-metabolizing enzymes CYP26A1 and CYP26B1 during murine organogenesis. Mechanisms of Development, 110, 173-177.

Alnouti, Y., \& Klaassen, C. D. (2008).Tissue distribution, ontogeny, and regulation of aldehyde dehydrogenase (Aldh) enzymes mRNA by prototypical microsomal enzyme inducers in mice. Toxicological Sciences, 101, 51-64.

Anderson, E. L., Baltus, A. E., Roepers-Gajadien, H. L., Hassold, T. J., de Rooij, D. G., van Pelt, A. M., et al. (2008). Stra8 and its inducer, retinoic acid, regulate meiotic initiation in both spermatogenesis and oogenesis in mice. Proceedings of the National Academy of Sciences of the United States of America, 105, 14976-14980.

Baltus, A. E., Menke, D. B., Hu, Y. C., Goodheart, M. L., Carpenter, A. E., de Rooij, D. G., et al. (2006). In germ cells of mouse embryonic ovaries, the decision to enter meiosis precedes premeiotic DNA replication. Nature Genetics, 38, 1430-1434.

Barrios, F., Filipponi, D., Campolo F., Gori, M., Bramucci, F., Pellegrini M., et al. (2012). SOHLH1 and SOHLH2 control Kit expression during postnatal male germ cell development. Journal of Cell Science, 125, 1455-1464.

Blume-Jensen, P., Jiang, G., Hyman, R., Lee, K. F, O'Gorman, S., \& Hunter, T. (2000). Kit/stem cell factor receptor-induced activation of phosphatidylinositol 3'-kinase is essential for male fertility. Nature Genetics, 24,157-162.

Bowles, J., Feng, C. W, Knight, D., Smith, C. A, Roeszler, K. N, Bagheri-Fam, S., et al. (2009). Malespecific expression of Aldh1a1 in mouse and chicken fetal testes: implications for retinoid balance in gonad development. Developmental Dynamics, 238, 2073-2080.

Bowles, J., Feng, C. W, Miles, K., Ineson, J., Spiller, C., \& Koopman, P. (2016). ALDH1A1 provides a source of meiosis-inducing retinoic acid in mouse fetal ovaries. Nature Communications, 7 , 10845.

Bowles, J., Feng, C. W, Spiller, C., Davidson, T. L, Jackson, A., \& Koopman, P. (2010). FGF9 suppresses meiosis and promotes male germ cell fate in mice. Developmental Cell, 19, 440449.

Bowles, J., Knight, D., Smith, C., Wilhelm, D., Richman, J., Mamiya, S., et al. (2006). Retinoid signaling determines germ cell fate in mice. Science, 312, 596-600.

Bowles, J., \& Koopman, P. (2007). Retinoic acid, meiosis and germ cell fate in mammals. Development, 134, 3401-3411. 
Buaas, F. W, Kirsh, A. L, Sharma, M., McLean, D. J, Morris, J. L, Griswold, M. D, et al. (2004). Plzf is required in adult male germ cells for stem cell self-renewal. Nature Genetics, 36, 647-652.

Chen, Y., Ma, L., Hogarth, C., Wei, G., Griswold, M. D., \& Tong, M. H. (2016). Retinoid signaling controls spermatogonial differentiation by regulating expression of replication-dependent core histone genes. Development, 143, 1502-1511.

Clermont, Y., \& Perey, B. (1957). Quantitative study of the cell population of the seminiferous tubules in immature rats. The American Journal of Anatomy, 100, 241-267.

Costoya, J. A, Hobbs, R. M, Barna, M., Cattoretti, G., Manova, K., Sukhwani, M., et al. (2004). Essential role of Plzf in maintenance of spermatogonial stem cells. Nature Genetics, 36, 653659.

Davis, J. C, Snyder, E. M, Hogarth, C. A, Small, C., \& Griswold, M. D. (2013). Induction of spermatogenic synchrony by retinoic acid in neonatal mice. Spermatogenesis, 3, e23180.

de Rooij, D. G, \& Russell, L. D. (2000). All you wanted to know about spermatogonia but were afraid to ask. Journal of Andrology, 21, 776-798.

de Rooij, DG. (1998). Stem cells in the testis. International Journal of Experimental Pathology, 79, 6780.

Drumond, A. L, Meistrich, M. L, \& Chiarini-Garcia, H. (2011). Spermatogonial morphology and kinetics during testis development in mice: a high-resolution light microscopy approach. Reproduction, $142,145-155$.

Duester, G. (2008). Retinoic acid synthesis and signaling during early organogenesis. Cell, 134, 921931.

Evans, E., Hogarth, C., Mitchell, D., \& Griswold, M. (2014). Riding the spermatogenic wave: profiling gene expression within neonatal germ and Sertoli cells during a synchronized initial wave of spermatogenesis in mice. Biology of Reproduction, 90, 1-12.

Fan, X., Molotkov, A., Manabe, S., Donmoyer, C. M., Deltour, L., Foglio, M. H., et al. (2003). Targeted disruption of Aldh1a1 (Raldh1) provides evidence for a complex mechanism of retinoic acid synthesis in the developing retina. Molecular and Cellular Biology, 23, 4637-4648.

Filipponi, D., Hobbs, R. M., Ottolenghi, S., Rossi, P., Jannini, E. A, Pandolfi P. P., et al. (2007). Repression of kit expression by Plzf in germ cells. Molecular and Cellular Biology, 27, 67706781.

Gaemers, I. C., van Pelt, A. M., van der Saag, P. T., \& de Rooij, D. G. (1996). All-trans-4-oxo-retinoic acid: a potent inducer of in vivo proliferation of growth-arrested $A$ spermatogonia in the vitamin A-deficient mouse testis. Endocrinology, 137, 479-485.

Gagnon, I., Duester, G., \& Bhat, P. V. (2002). Kinetic analysis of mouse retinal dehydrogenase type-2 (RALDH2) for retinal substrates. Biochimica et Biophysica Acta, 1596, 156-162.

Gely-Pernot, A., Raverdeau, M., Célébi, C., Dennefeld, C., Feret, B., Klopfenstein, M., et al. (2012).Spermatogonia differentiation requires retinoic acid receptor gamma. Endocrinology, 153, 438-449.

Gely-Pernot, A., Raverdeau, M., Teletin, M., Vernet, N., Féret, B., Klopfenstein, M., et al. (2015). Retinoic acid receptors control spermatogonia cell-fate and induce expression of the SALL4A transcription factor. PLoS Genetics, 11, e1005501.

Germain, P., Gaudon, C., Pogenberg, V., Sanglier, S., Van Dorsselaer, A., Royer, C. A., et al. (2009). Differential action on coregulatory interaction defines inverse retinoid agonists and neutral antagonists. Chemistry \& Biology, 16, 479-489.

Ghyselinck, N.B, Vernet, N., Dennefeld C., Giese N., Nau H., Chambon P., et al. (2006). Retinoids and spermatogenesis: lessons from mutant mice lacking the plasma retinol binding protein. Developmental Dynamics, 235, 1608-1622.

Griswold, M. D. (2016). Spermatogenesis: The Commitment to meiosis. Physiological Reviews, 96, 117. 
Griswold, M. D., Hogarth, C. A., Bowles, J., \& Koopman, P. (2012). Initiating meiosis: the case for retinoic acid. Biology of Reproduction, 86, 35.

Hara, K., Nakagawa, T., Enomoto, H., Suzuki, M., Yamamoto, M., Simons, B. D., et al. (2014). Mouse spermatogenic stem cells continually interconvert between equipotent singly isolated and syncytial states. Cell Stem Cell, 14, 658-672.

Hobbs R. M., Fagoonee S., Papa A., Webster, K., Altruda, F., Nishinakamura, R., et al. (2012). Functional antagonism between Sall4 and Plzf defines germline progenitors. Cell Stem Cell, 10, 284-298.

Hogarth, C. A, Evanoff, R., Mitchell, D., Kent, T., Small, C., Amory, J. K, et al. (2013). Turning a spermatogenic wave into a tsunami: synchronizing murine spermatogenesis using WIN 18,446. Biology of Reproduction, 88, 40.

Hogarth, C. A., Arnold, S., Kent, T., Mitchell, D., Isoherranen, N., \& Griswold M. D. (2015a). Processive pulses of retinoic acid propel asynchronous and continuous murine sperm production. Biology of Reproduction, 92, 37.

Hogarth, C. A., Evans, E., Onken, J., Kent, T., Mitchell, D., Petkovich, M., et al. (2015b). CYP26 enzymes are necessary within the postnatal seminiferous epithelium for normal murine spermatogenesis. Biology of Reproduction, 93, 19.

Horton, C., \& Maden, M. (1995). Endogenous distribution of retinoids during normal development and teratogenesis in the mouse embryo. Developmental Dynamics, 202, 312-323.

Ikami, K., Tokue, M., Sugimoto, R., Noda, C., Kobayashi, S., Hara, K., et al. (2015). Hierarchical differentiation competence in response to retinoic acid ensures stem cell maintenance during mouse spermatogenesis. Development, 142, 1582-1592.

Jørgensen, A., Nielsen, J. E., Perlman, S., Lundvall, L., Mitchell, R. T., Juul, A., \& Rajpert-De Meyts, E. (2015). Ex vivo culture of human fetal gonads: manipulation of meiosis signalling by retinoic acid treatment disrupts testis development. Human Reproduction, 30, 2351-2363.

Kane, M. A., Folias, A. E., Wang, C., \& Napoli, J. L. (2008). Quantitative profiling of endogenous retinoic acid in vivo and in vitro by tandem mass spectrometry. Analytical Chemistry, 80, 1702 1708.

Kawai, T., Yanaka, N., Richards, J. S., \& Shimada, M. (2016). De novo-synthesized retinoic acid in ovarian antral follicles enhances FSH-mediated ovarian follicular cell differentiation and female fertility. Endocrinology, 157, 2160-2172.

Kent, T., Arnold, S. L., Fasnacht, R., Rowsey, R., Mitchell, D., Hogarth, C. A., et al. (2016). ALDH enzyme expression is independent of the spermatogenic cycle, and their inhibition causes misregulation of murine spermatogenic processes. Biology of Reproduction, 94, 12.

Kissel, H. I., Timokhina, M. P., Hardy, G., Rothschild, Y., Tajima, V., Soares, M., et al. (2000). Point mutation in kit receptor tyrosine kinase reveals essential roles for kit signaling in spermatogenesis and oogenesis without affecting other kit responses. The EMBO Journal, 19, 1312-1326.

Kluin, P. M., Kramer, M. F., \& de Rooij, D. G. (1982). Spermatogenesis in the immature mouse proceeds faster than in the adult. International Journal of Andrology, 5, 282-294.

Kocer, A., Reichmann, J., Best, D., \& Adams, I. R. (2009). Germ cell sex determination in mammals. Molecular Human Reproduction, 15, 205-213.

Koshimizu, U., Watanabe, M., \& Nakatsuji, N. (1995). Retinoic acid is a potent growth activator of mouse primordial germ cells in vitro. Developmental Biology, 168, 683-685.

Koubova, J., Menke, D. B., Zhou, Q., Capel, B., Griswold, M. D., \& Page, D. C. (2006). Retinoic acid regulates sex-specific timing of meiotic initiation in mice. Proceedings of the National Academy of Sciences of the United States of America, 103, 2474-2479.

Kumar, S., Chatzi, C., Brade, T., Cunningham, T. J., Zhao, X., \& Duester, G. (2011). Sex-specific timing of meiotic initiation is regulated by Cyp26b1 independent of retinoic acid signalling. Nature Communications, 2, 151. 
Le Bouffant, R., Guerquin, M. J., Duquenne, C., Frydman, N., Coffigny, H., Rouiller-Fabre, V., et al. (2010). Meiosis initiation in the human ovary requires intrinsic retinoic acid synthesis. Human Reproduction, 25, 2579-2590.

Li, H., MacLean, G., Cameron, D., Clagett-Dame, M., \& Petkovich, M. (2009). Cyp26b1 expression in murine Sertoli cells is required to maintain male germ cells in an undifferentiated state during embryogenesis. PLoS One, 4, e7501.

MacLean, G., Li, H., Metzger, D., Chambon, P., \& Petkovich, M. (2007). Apoptotic extinction of germ cells in testes of Cyp26b1 knockout mice. Endocrinology, 148, 4560-4567.

Mark, M., Ghyselinck, N. B., \& Chambon, P. (2006). Function of retinoid nuclear receptors: lessons from genetic and pharmacological dissections of the retinoic acid signaling pathway during mouse embryogenesis Annual Review of Pharmacology and Toxicology, 46, 451-80.

Mark, M., Jacobs, H., Oulad-Abdelghani, M., Dennefeld, C., Féret, B., Vernet, N., et al. (2008). STRA8-deficient spermatocytes initiate, but fail to complete, meiosis and undergo premature chromosome condensation. Journal of Cell Science, 121, 3233-3242.

Mark, M., Teletin, M., Vernet, N., \& Ghyselinck, N.B. (2015). Role of retinoic acid receptor (RAR) signaling in post-natal male germ cell differentiation. Biochimica et Biophysica Acta. 1849, 84 93.

Matt, N., Dupé, V., Garnier, J. M., Dennefeld, C., Chambon, P., Mark, M., et al. (2005). Retinoic aciddependent eye morphogenesis is orchestrated by neural crest cells. Development, 132, 47894800.

McLaren, A., \& Lawson, K. A. (2005). How is the mouse germ-cell lineage established? Differentiation, $73,435-437$.

Mendelsohn, C., Lohnes, D., Decimo, D., Lufkin, T., LeMeur, M., Chambon, P., \& Mark, M. (1994). Function of the retinoic acid receptors (RARs) during development (II). Multiple abnormalities at various stages of organogenesis in RAR double mutants. Development, 120, 2749-2771.

Morales, C., \& Griswold, M. D. (1987). Retinol-induced stage synchronization in seminiferous tubules of the rat. Endocrinology, 121, 432-434.

Morita, Y., \& Tilly, J. L. (1999). Segregation of retinoic acid effects on fetal ovarian germ cell mitosis versus apoptosis by requirement for new macromolecular synthesis. Endocrinology, 140, 26962703.

Nagano R., Tabata S., Nakanishi Y., Ohsako S., Kurohmaru M., \& Hayashi Y. (2000). Reproliferation and relocation of mouse male germ cells (gonocytes) during prespermatogenesis. The Anatomical Record, 258, 210-220.

Nakata, H., Wakayama, T., Sonomura, T., Honma, S., Hatta, T., \& Iseki, S. (2015). Three-dimensional structure of seminiferous tubules in the adult mouse. Journal of Anatomy, 227, 686-694.

Niederreither, K., McCaffery, P., Dräger, U. C, Chambon, P., \& Dollé, P. (1997) Restricted expression and retinoic acid-induced downregulation of the retinaldehyde dehydrogenase type 2 (RALDH2) gene during mouse development. Mechanisms of Development, 62, 67-78.

Niederreither, K., Vermot, J., Fraulob, V., Chambon, P., \& Dollé, P. (2002). Retinaldehyde dehydrogenase 2 (RALDH2)- independent patterns of retinoic acid synthesis in the mouse embryo. Proceedings of the National Academy of Sciences of the United States of America, 99, 16111-16116.

Novák, J., Benísek, M., \& Hilscherová, K. (2008). Disruption of retinoid transport, metabolism and signaling by environmental pollutants. Environment International, 34, 898-913.

Oulad-Abdelghani, M., Bouillet, P., Decimo, D., Gansmuller, A., Heyberger, S., Dollé, P., et al. (1996). Characterization of a premeiotic germ cell-specific cytoplasmic protein encoded by Stra8, a novel retinoic acid-responsive gene. The Journal of Cell Biology, 135, 469-477.

Piprek, R. P., Pecio, A., Laskowska-Kaszub, K, Kloc, M, Kubiak, J. Z., \& Szymura, J. M. (2013). Retinoic acid homeostasis regulates meiotic entry in developing anuran gonads and in Bidder's organ through Raldh2 and Cyp26b1 proteins. Mechanisms of Development, 130, 613-627. 
Raverdeau, M., Gely-Pernot, A., Féret, B., Dennefeld, C., Benoit, G., Davidson, I., et al. (2012). Retinoic acid induces Sertoli cell paracrine signals for spermatogonia differentiation but cell autonomously drives spermatocyte meiosis. Proceedings of the National Academy of Sciences of the United States of America, 109, 16582-16587.

Rossant, J., Zirngibl, R., Cado, D., Shago, M., \& Giguère, V. (1991). Expression of a retinoic acid response element-hsplacZ transgene defines specific domains of transcriptional activity during mouse embryogenesis. Genes \& Development, 5, 1333-1344.

Russell, L. D., Ettlin, A. P. S., \& Clegg, R. A. (1990). Histological and histopathological evaluation of the testis. Clearwater, FL: Cache River Press.

Samarut, E., \& Rochette-Egly, C. (2012). Nuclear retinoic acid receptors: conductors of the retinoic acid symphony during development. Molecular and Cellular Endocrinology, 348, 348-360.

Sarath Josh, M. K., Pradeep, S., Vijayalekshmi Amma, K. S., Balachandran, S., Abdul Jaleel, U. C., Doble, M., et al. (2014). Phthalates efficiently bind to human peroxisome proliferator activated receptor and retinoid $X$ receptor $\alpha, \beta$, y subtypes: an in silico approach. Journal of Applied Toxicology, 34, 754-765.

Schrans-Stassen, B. H., van de Kant, H. J., de Rooij, D. G., \& van Pelt, A. M. (1999). Differential expression of kit in mouse undifferentiated and differentiating type A spermatogonia. Endocrinology, 140, 5894-5900.

Shinohara, T., Orwig, K. E., Avarbock, M. R., \& Brinster, R. L. (2000). Spermatogonial stem cell enrichment by multiparameter selection of mouse testis cells. Proceedings of the National Academy of Sciences of the United States of America, 97, 8346-8351.

Snyder, E. M., Davis, J. C., Zhou, Q., Evanoff, R., \& Griswold, M. D. (2011). Exposure to retinoic acid in the neonatal but not adult mouse results in synchronous spermatogenesis. Biology of Reproduction, 84, 886-893.

Soone, S. B., Almstrup, K., Dalgaard, M., Juncker, A. S., Edsgard, D., Ruban, L., et al. (2009). Analysis of gene expression profiles of microdissected cell populations indicates that testicular carcinoma in situ is an arrested gonocyte. Cancer Research, 69, 5241-5250.

Sugimoto, R., Nabeshima, Y., \& Yoshida, S. (2012). Retinoic acid metabolism links the periodical differentiation of germ cells with the cycle of Sertoli cells in mouse seminiferous epithelium. Mechanisms of Development, 128, 610-624.

Tedesco, M., Desimio, M. G., Klinger, F. G., De Felici, M., \& Farini, D. (2013). Minimal concentrations of retinoic acid induce stimulation by retinoic acid 8 and promote entry into meiosis in isolated pregonadal and gonadal mouse primordial germ cells. Biology of Reproduction, 88, 145.

Tong, M. H, Yang, Q. E., Davis, J. C., \& Griswold, M. D. (2013). Retinol dehydrogenase 10 is indispensable for spermatogenesis in juvenile males. Proceedings of the National Academy of Sciences of the United States of America, 110, 543-548.

Trautmann, E., Guerquin, M.J., Duquenne, C, Lahaye, J.B., Habert, R., \& Livera G. (2008). Retinoic acid prevents germ cell mitotic arrest in mouse fetal testes. Cell Cycle, 7, 656-664.

Van Beek, M. E., \& Meistrich, M. L. (1990). A method for quantifying synchrony in testes of rats treated with vitamin A deprivation and readministration. Biology of Reproduction, 42, 424-431.

van Pelt, A. M., \& de Rooij, D. G. (1990). The origin of the synchronization of the seminiferous epithelium in vitamin A-deficient rats after vitamin A replacement. Biology of Reproduction, 42, 677-682.

van Pelt, A. M., \& de Rooij, D. G. (1991). Retinoic acid is able to reinitiate spermatogenesis in vitamin A-deficient rats and high replicate doses support the full development of spermatogenic cells. Endocrinology, 128, 697-704.

van Pelt, A. M., van Dissel-Emiliani, F. M., Gaemers, I. C., van der Burg, M. J., Tanke, H. J., \& de Rooij D. G. (1995). Characteristics of A spermatogonia and preleptotene spermatocytes in the vitamin A-deficient rat testis. Biology of Reproduction, 53, 570-578. 
Vernet N., Dennefeld C., Rochette-Egly C., Oulad-Abdelghani M., Chambon P., Ghyselinck N. B., et al. (2006a). Retinoic acid metabolism and signaling pathways in the adult and developing mouse testis. Endocrinology, 147, 96-110.

Vernet, N., Dennefeld, C., Guillou, F., Chambon, P., Ghyselinck, N. B., \& Mark, M. (2006b). Prepubertal testis development relies on retinoic acid but not rexinoid receptors in Sertoli cells. The EMBO Journal, 25, 5816-5825.

Wallacides, A., Chesnel, A., Chardard, D., Flament, S., \& Dumond, H. (2009). Evidence for a conserved role of retinoic acid in urodele amphibian meiosis onset. Developmental Dynamics, 238, 1389-1398.

Western, P.S., Miles, D.C., van den Bergen, J.A., Burton, M., \& Sinclair, A. H. (2008). Dynamic regulation of mitotic arrest in fetal male germ cells. Stem Cells, 26, 339-347.

Wolbach, S. B., \& Howe, P. R. (1925). Tissue changes following deprivation of fat-soluble A vitamin. The Journal of Experimental Medicine, 42, 753-777.

Wu, X., Schmidt, J. A., Avarbock, M. R., Tobias, J. W., Carlson, C. A., Kolon, T. F., et al. (2009). Prepubertal human spermatogonia and mouse gonocytes share conserved gene expression of germline stem cell regulatory molecules. Proceedings of the National Academy of Sciences, 106, 21672-21677.

Wu, J. W., Wang, R. Y., Guo, Q. S., \& Xu, C. (2008). Expression of the retinoic acid-metabolizing enzymes RALDH2 and CYP26b1 during mouse postnatal testis development. Asian Journal of Andrology, 10, 569-576.

Yoshida, S., Sukeno, M., Nakagawa, T., Ohbo, K., Nagamatsu, G., Suda, T., et al. (2006). The first round of mouse spermatogenesis is a distinctive program that lacks the self-renewing spermatogonia stage. Development, 133, 1495-1505.

Zhou, Q., Li, Y., Nie, R., Friel, P., Mitchell, D., Evanoff, R. M., et al. (2008). Expression of stimulated by retinoic acid gene 8 (Stra8) and maturation of murine gonocytes and spermatogonia induced by retinoic acid in vitro. Biology of Reproduction, 78, 537-545. 


\section{Figures}

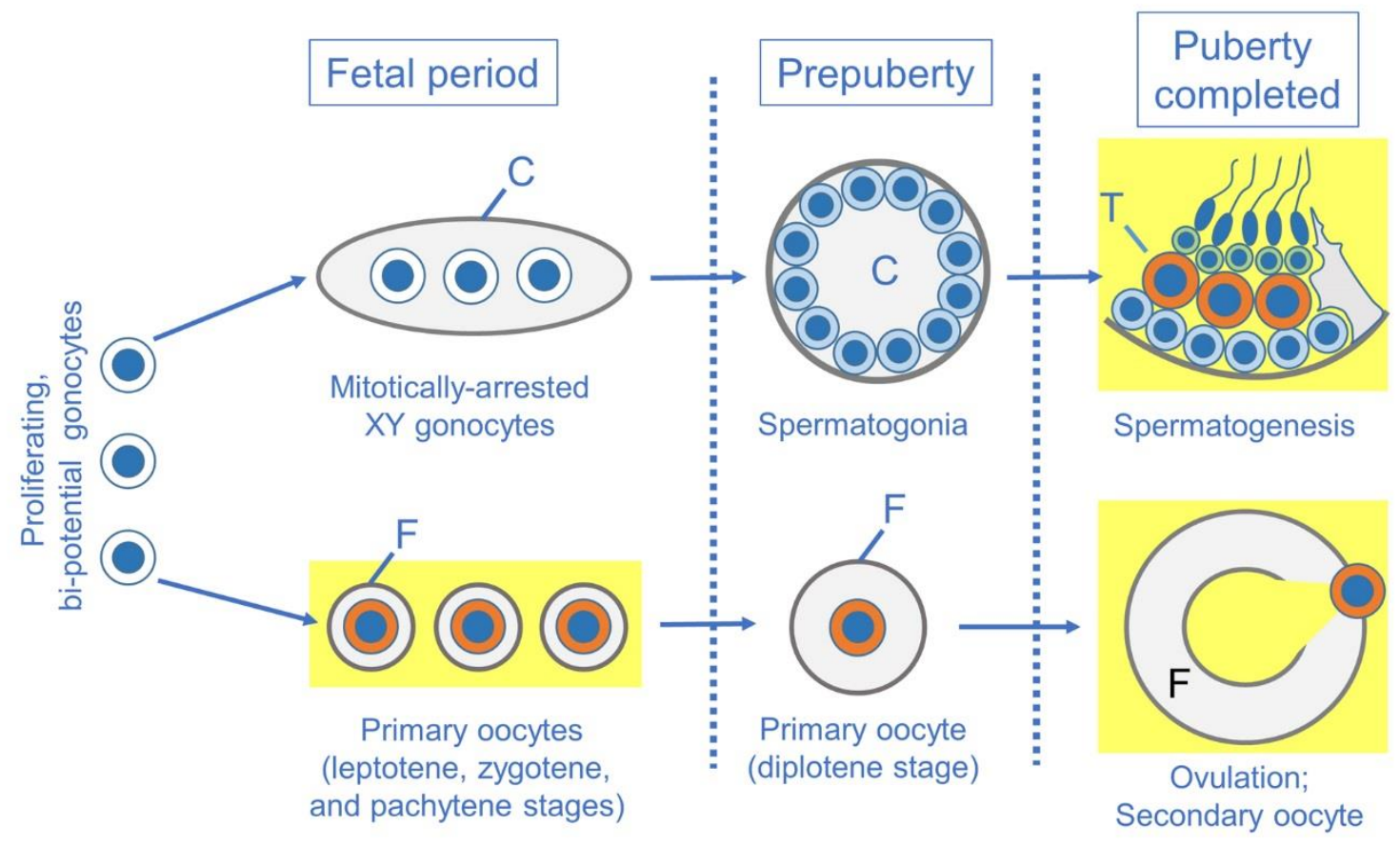

Figure 1. Key stages of fetal and postnatal germ cell development. Fetal period: female gonocytes enter meiosis prior to birth, while male gonocytes undergo a mitotic cell cycle arrest. Prepuberty: during the first postnatal week, male gonocytes resume proliferation and migrate from the center of the seminiferous cords to the basement membrane, where they generate spermatogonia. Oogenesis and spermatogenesis are completed at puberty. Meiotic cells (oocytes or spermatocytes) and male post-meiotic cells (spermatids) are in orange and green, respectively. Supporting somatic cells (follicular or Sertoli cells) are represented in grey. Legend: $\mathrm{C}$ and T, seminiferous cords and tubules, respectively; $F$, ovarian follicles. Processes requiring ATRA are drawn on a yellow background. See the main text for details. 


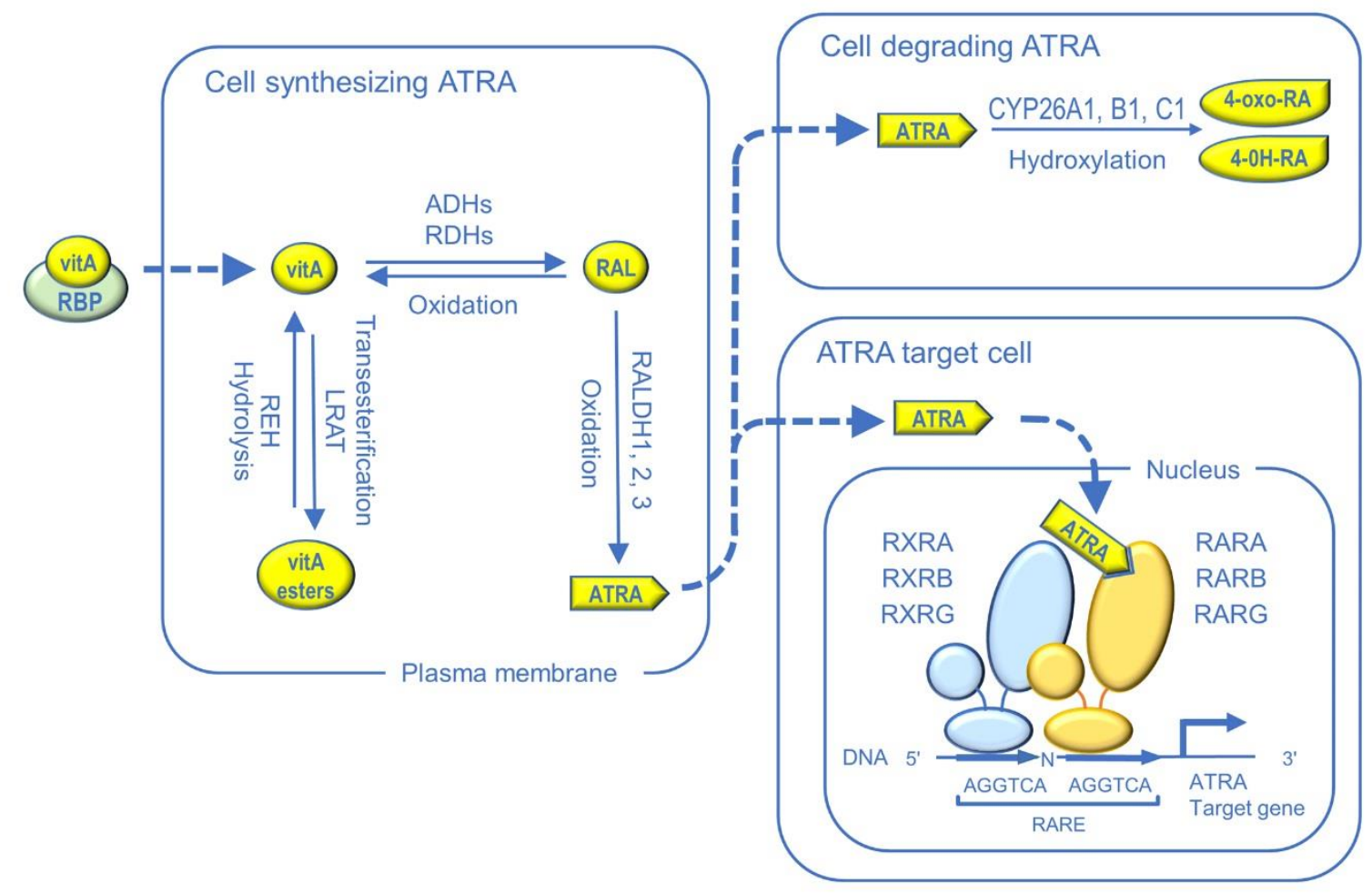

Figure 2. Regulation of retinoid signaling. Vitamin $A$ (vitA) is provided to cells either by the blood, where it circulates bound to the vitA-binding protein (RBP). In cells, vitA is locally stored in the form of vitA esters generated through the transesterification activity of LRAT (lecithin retinol acyltransferase, the most potent vitA-esterifying enzyme). VitA is liberated from vitA-ester stores through the hydrolyzing activity of retinyl ester hydrolases (REH). In ATRA synthesizing cells, vitA is oxidized into retinaldehyde (RAL) by alcohol dehydrogenases (ADH) and short chain retinol dehydrogenases (RDH). RAL dehydrogenases (RALDH1, 2 and/or 3) then generate ATRA, which binds within the nucleus to a RAR forming a heterodimer complex with a RXR. RAR/RXR modulate transcription by binding to DNA at RARE motifs located in regulatory regions of ATRA target-genes. In cells expressing CYP26A1, B1 and/or C1 enzymes, ATRA is hydroxylated into polar compounds such as 4-oxo- and 4-hydroxyretinoic acid (4-oxo-RA and 4-OH-RA, respectively), which are subject to further metabolism and elimination. ATRA often acts in a paracrine manner on neighboring cells, but there also is evidence for autocrine effects in cells that synthesize it. Dashed line indicate diffusion of retinoids through cell membranes. See the main text for details. 

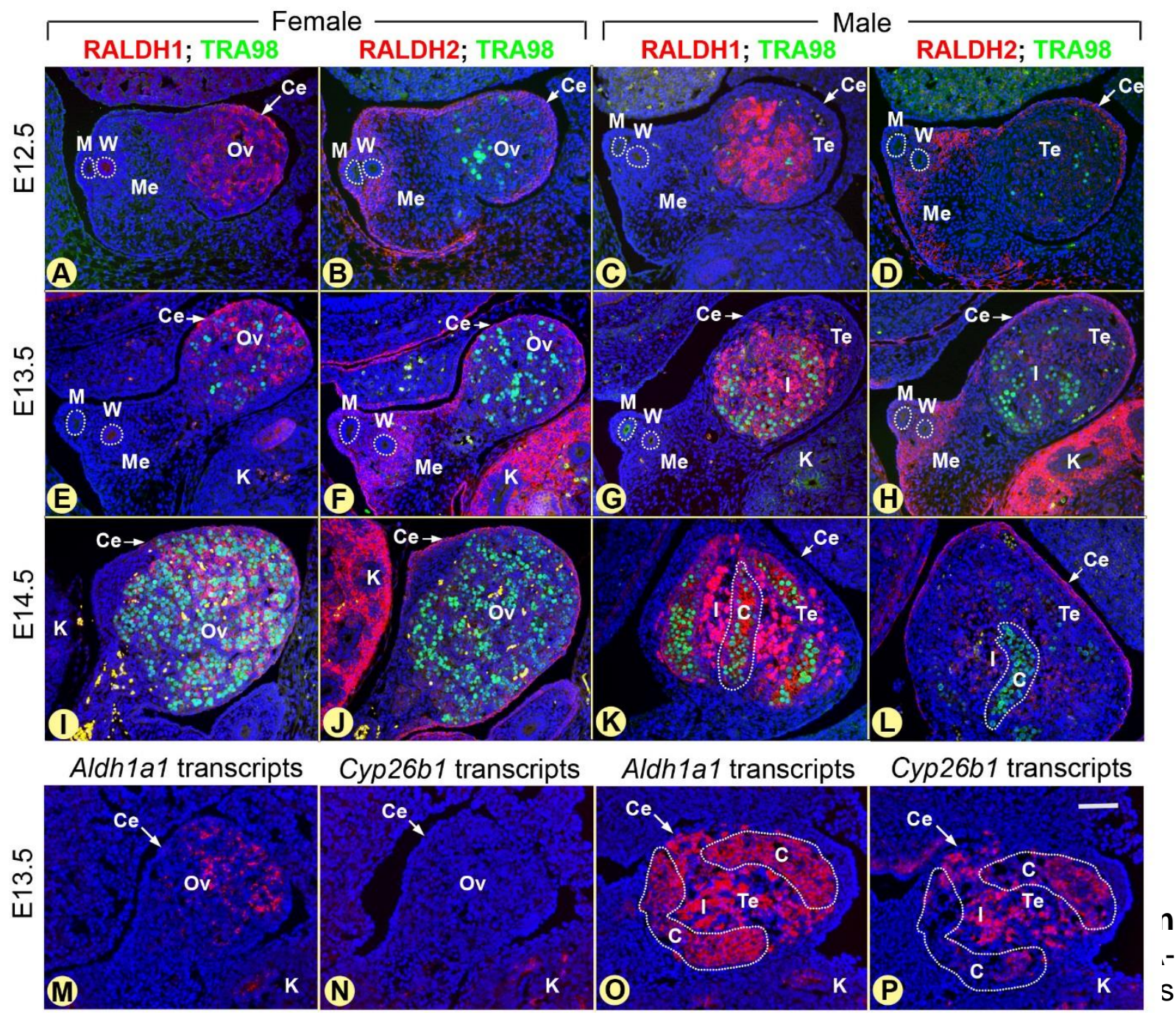

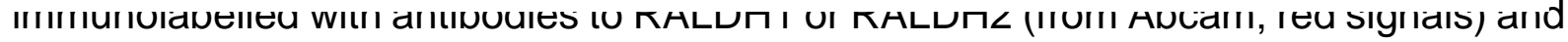
to TRA98 (from Abcam), a nuclear protein specifically expressed in (some) gonocytes (green signals). The sections were counterstained with 4',6-diamidino-2-phenylindole (DAPI; blue signal) to label cell nuclei. RALDH1 and RALDH2, are restricted to the somatic cells located in the gonad (ovary, Ov; testis, Te), the coelomic epithelium (Ce) and the mesonephros (Me). Gonocytes, either positive or negative for TRA98, never express any of the two RALDH. (M-P) Adjacent $(M, N)$ or consecutive $(O, P)$ sections hybridized with anti-sense probes to Aldh1a1 and Cyp26b1 as described (Vernet et al., 2006a). Note that some of the immature Sertoli cells within the nascent testis cords express both transcripts. The ISH signals were converted to a red color and the histological sections were counterstained with DAPI. K, (definitive) kidney; M, Müllerian duct; W, Wolffian duct. Bar in P: $160 \mu \mathrm{m}(\mathrm{A}-\mathrm{P})$. 

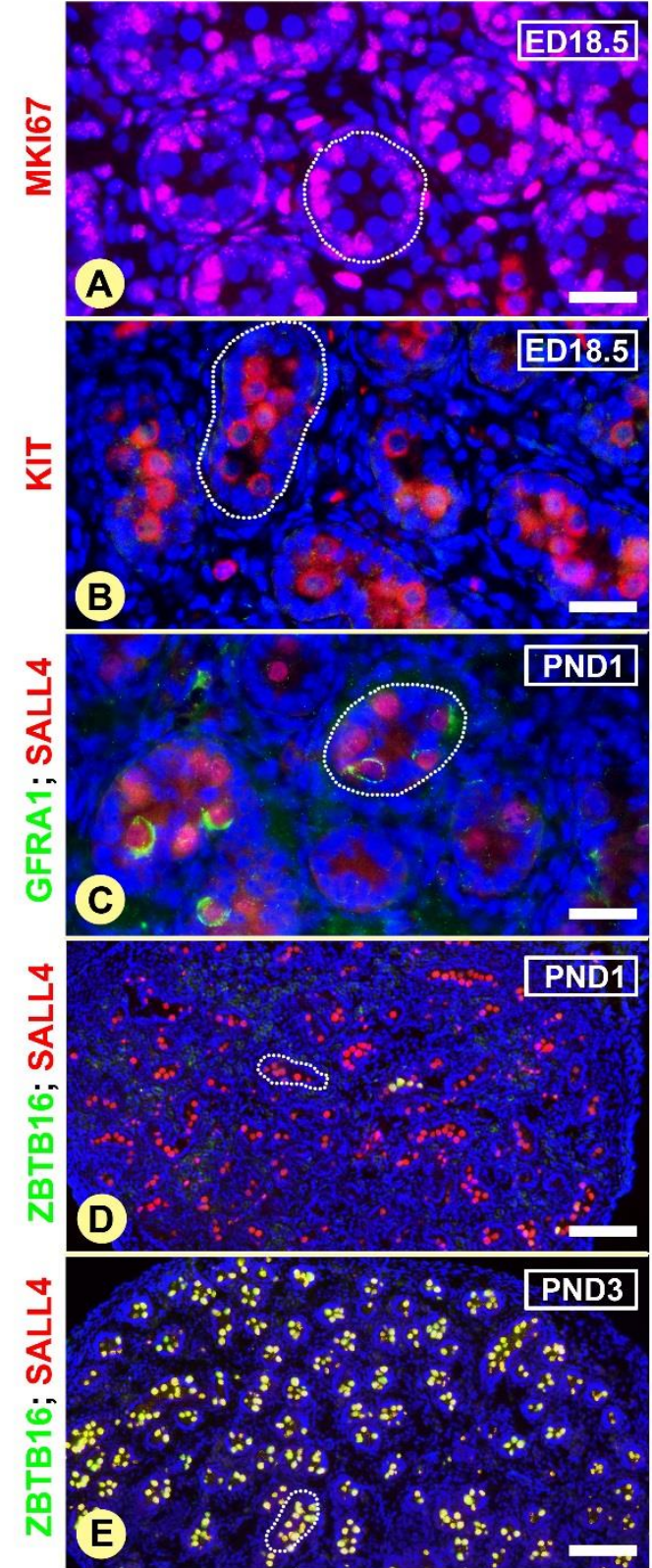
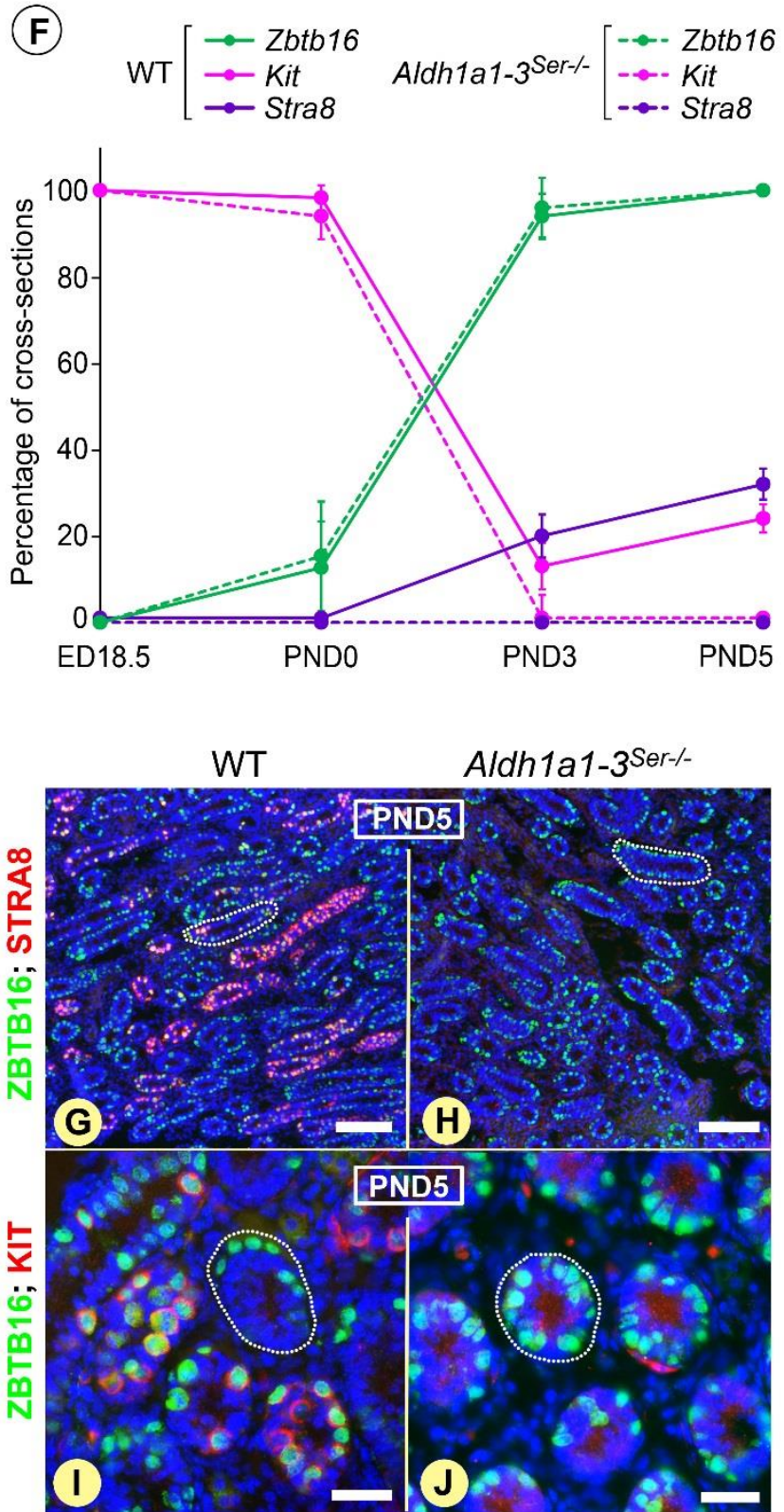

Figure 4. The transition from gonocytes to differentiating spermatogonia, but not the formation of undifferentiated spermatogonia, is impaired in the absence of ATRA in the seminiferous epithelium. (A, B) In both Aldh1a1-35er-/ and WT mice at ED18.5, the proliferation marker MKI67 (red signal, A) is strictly confined to the somatic cells, including immature Sertoli cells at the periphery of the seminiferous cords (dotted white line), while expression of KIT (B, red signal) is identical in GC. (C-E) Between ED18.5 and PND3, expression of GFRA1 (C, green signal) and ZBTB16 (D and E, green and yellow signals) also is identical in GC of both Aldh1a1-3 $3^{\text {Ser-/- }}$ and WT mice. (F) Percentage of cross-sections of seminiferous cords harboring GC expressing: ZBTB16 (green lines), KIT (pink lines) and STRA8 (purple lines) in WT (solid lines) and Aldh1a1-3 ${ }^{\text {Ser-- }}$ (dotted lines) mice, at the indicated ages. The bars represent mean \pm SEM ( $n=3$ mice, $>200$ cross sections per mice). (G-J) STRA8 and KIT (red signals) are not expressed in spermatogonia from Aldh1a1-3 $3^{\text {Ser-- }}$ testes $(\mathrm{H}, \mathrm{J})$, in contrast to the WT situation (G, I). Scale bars: $30 \mu \mathrm{m}(\mathrm{A}-\mathrm{C}, \mathrm{H}, \mathrm{I})$ and $160 \mu \mathrm{m}(\mathrm{D}-\mathrm{G})$. 


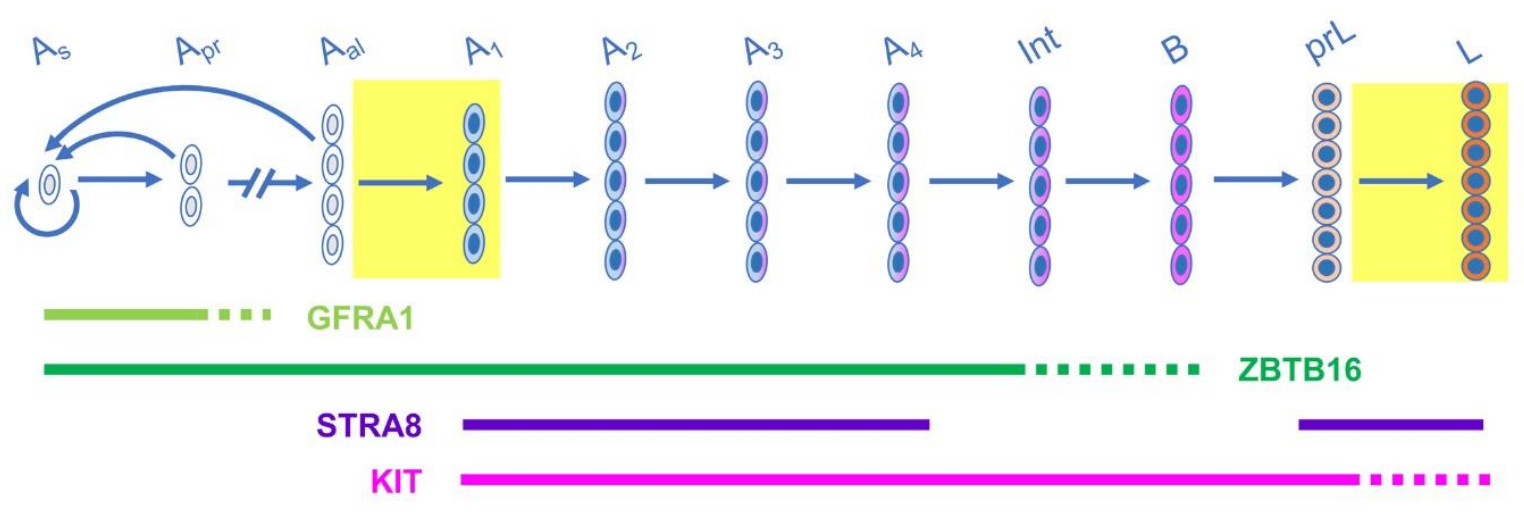

Figure 5. Spermatogonia differentiation assessed by immunohistochemistry. Because identification of spermatogonia subtypes at the morphological level can only rely on differences in nuclear shape and heterochromatin appearance that are both subtle and variable, immunohistochemical assays represent a valuable alternative. GFRA1 (light green line) is strongly expressed at the surface of $A_{\text {single }}\left(A_{s}\right)$ and $A_{\text {paired }}$ $\left(A_{p r}\right)$, and faintly in $A_{\text {aligned-4 }}\left(A_{a l-4}\right)$ spermatogonia (Gely-Pernot et al., 2012). ZBTB16, a nuclear protein (dark green line), is frequently considered as a marker Aundiff spermatogonia, as it is required for the maintenance of the spermatogonia stem cell pool (Buaas et al., 2004; Costoya et al., 2004). However, it is actually also strongly expressed in $\mathrm{A}_{1}-\mathrm{A}_{4}$ spermatogonia and, albeit at low levels, also in intermediary (int) and $B$ spermatogonia (our personal observations). (iii) STRA8 (purple line), is expressed in $\mathrm{A}_{1}-\mathrm{A}_{4}$ spermatogonia, absent in Int and $\mathrm{B}$ spermatogonia and reexpressed in preleptotene (prL) and leptotene (L) spermatocytes (Mark, Teletin, Vernet, \& Ghyselinck, 2015). Expression of the tyrosine kinase receptor KIT (pink line) is essential to the $\mathrm{Aal}_{\mathrm{al}} \mathrm{A}_{1}$ transition (Schrans-Stassen et al., 1999; Kissel et al, 2000; Blume-Jensen et al., 2000) and is restricted to differentiating ( $A_{1}$ to $\left.B\right)$ spermatogonia and prL spermatocytes (our observations). Note that: (i) the topological arrangement of $A_{s}, A_{p r}$, and $A_{a l}$ spermatogonia can only be visualized in whole mounts of seminiferous tubules; (ii) there are currently no marker that allow $A_{1}-A_{4}$ spermatogonia to be distinguished from one-another without knowing the stage of the SE cycle within which they reside. Cellular event requiring ATRA are on a yellow background. 


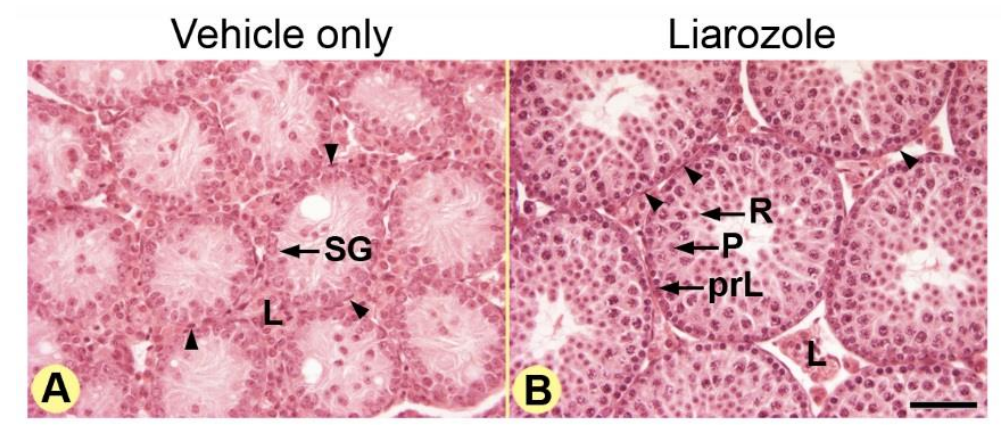

Figure 6. Pharmacological inhibition of CYP26 enzymes triggers spermatogenesis in a seminiferous epithelium that lacks all ATRA synthetic

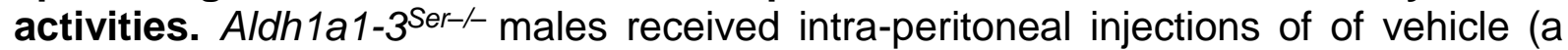
mixture of sunflower oil and ethanol) or liarozole $(40 \mathrm{mg} / \mathrm{kg}$ ) for 3 consecutive days at PNW3 and were sacrificed at PNW6. Testis section were stained with hematoxylin eosin. (A) After administration of the vehicle only, spermatogenesis remains blocked at the spermatogonia stage. (B) After treatment by liarozole, spermatogenesis progresses synchronously throughout the testis, to round spermatids. Legend: prL and $\mathrm{P}$, preleptotene and pachytene spermatocytes, respectively; L, Leydig cells; R, round spermatids; SG, spermatogonia. Arrowheads point to nuclei of peritubular myoid cells. Bar in B: $80 \mu \mathrm{m}$ (A and B).

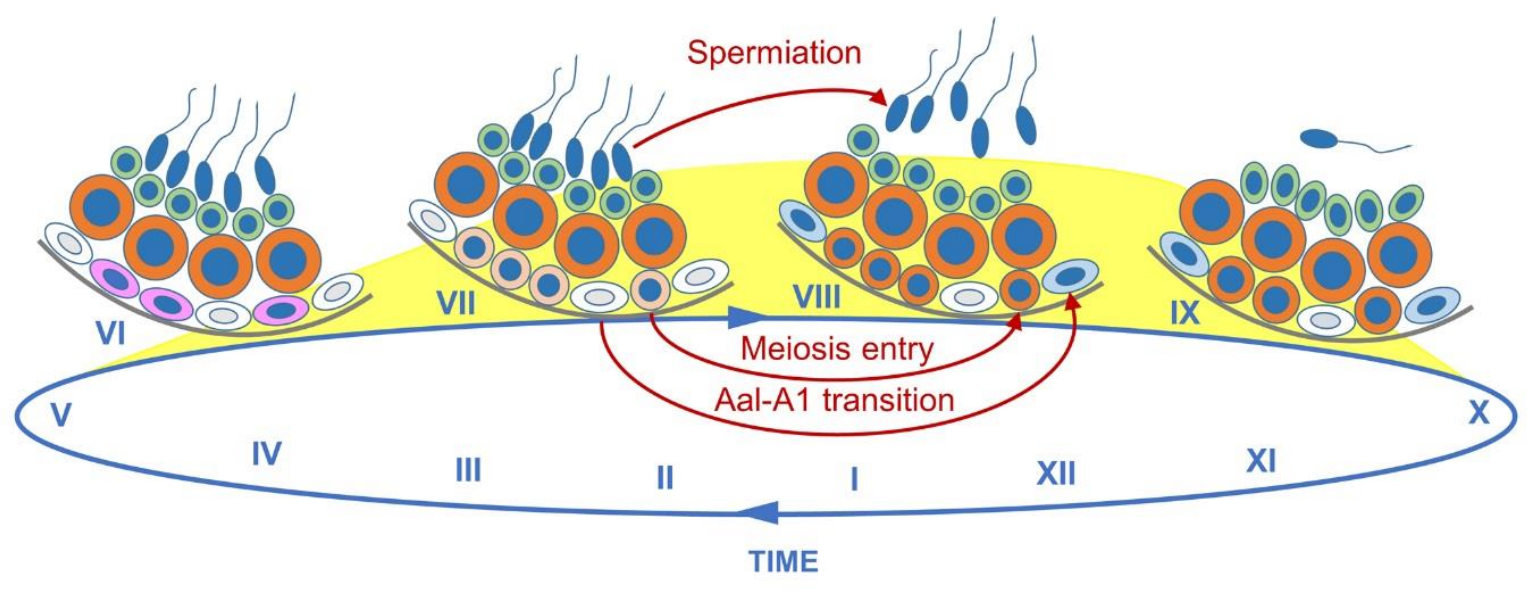

Figure 7. Diagram depicting the organization of the murine SE and the cellular associations (stages of the SE) present at the time of the ATRA pulse (in yellow). GC are organized in a stratified fashion from the periphery to the lumen of the seminiferous epithelium, forming three to four distinct layers. The most peripheral layer, in contact with the basement membrane, contains spermatogonia (white, Aal; blue, $A_{1}$; pink, B) and preleptotene spermatocytes (light orange), while more advanced spermatocytes (dark orange) are progressively displaced in the intermediate layer and post-meiotic cells (green, round, and blue, elongating, spermatids) reside in the layers closest to the lumen of the tubules. Roman numerals designate the 12 stages of the SE cycle. Cellular processes promoted by the rise in ATRA concentrations in the SE are indicated by the red arrows. For further details see the main text. 


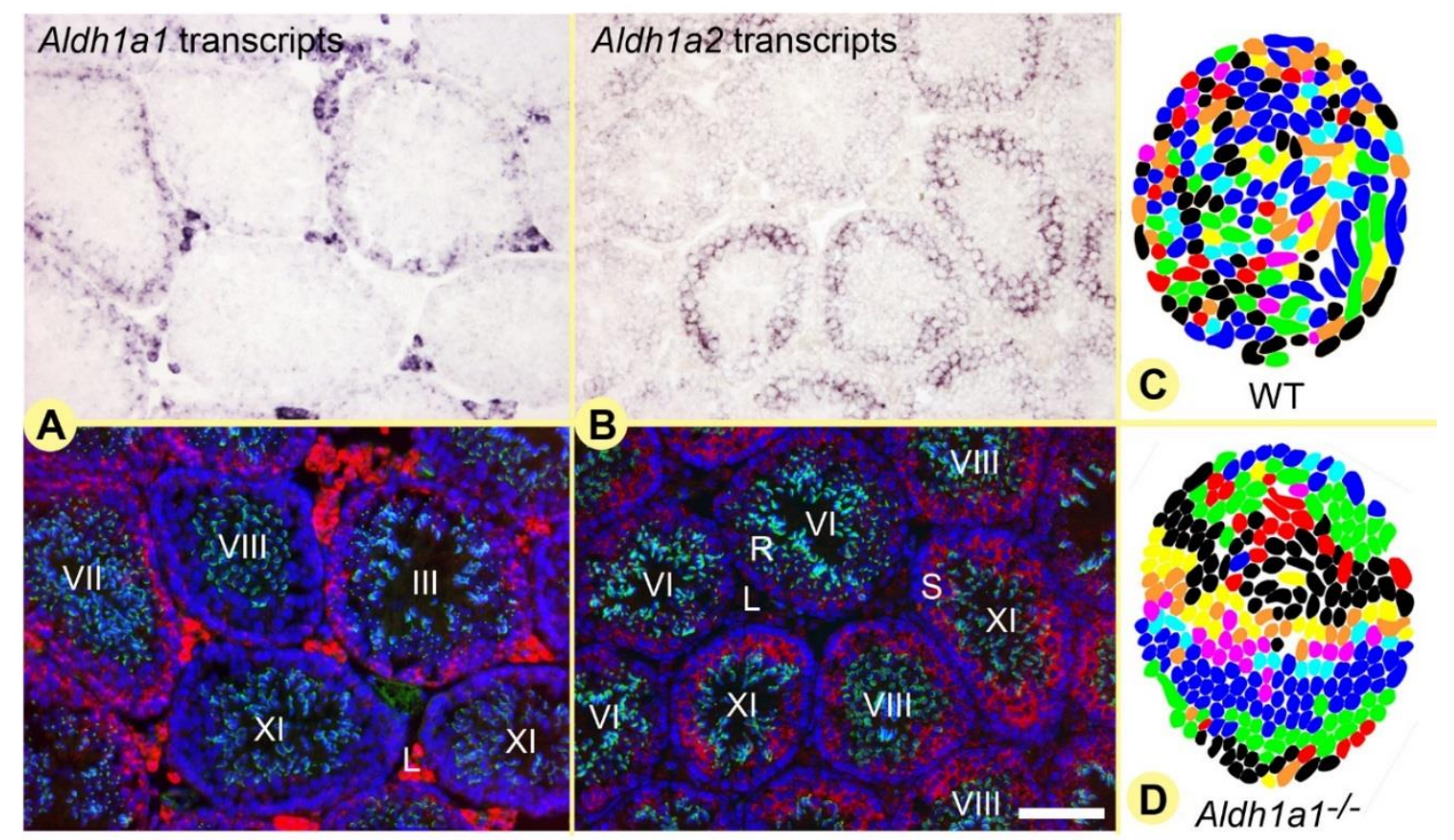

Figure 8. Roles for ATRA-synthesizing enzymes in regulating the SE cycle and wave. (A) In situ hybridization (ISH) using an anti-sense probe to Aldh1a1: higher mRNA levels (purple signals) are detected in Sertoli cells at stages II-VII of the SE cycle, as compared to stages VIII-XII and I. (B) ISH using an anti-sense probe to Aldh1a2: high mRNA levels are detected in spermatocytes at the late pachytene and diplotene stages (present at epithelial stages VIII to XII), as compared to spermatocytes present at stages I-VI; a weak ISH signal is also detected in round spermatids at stages II-VI. In the lower panels of (A) and (B), the ISH signals were converted to a red color, the histological sections were counterstained with $4^{\prime}, 6$ diamidino-2-phenylindole (blue signal) to label nuclei, and with Alexa Fluor 488conjugated peanut agglutinin (green signal) to label acrosomal systems. Epithelial stages were defined according to Russell, Ettlin, \& Clegg (1990), taking into consideration the shape of acrosomes (green signal). Roman numerals designate stages of the seminiferous epithelium cycle. Abbreviations: L, Leydig cells; R, round spermatids; S, spermatocytes. (C, D) transverse histological sections though testes of (C) WT and (D) Aldh1a1-/ mutants (see the main text for further details). Each colored spot represents a cross-section of a seminiferous tubule. Stages of the SE or group of stages are indicated in different colors: black, stages I-III; red, stage IV; green, stages $\mathrm{V}$ and $\mathrm{VI}$; dark blue, stages VII and VIIII; light blue, stage IX; pink, stage X; orange stage XI; yellow, stage XII. 


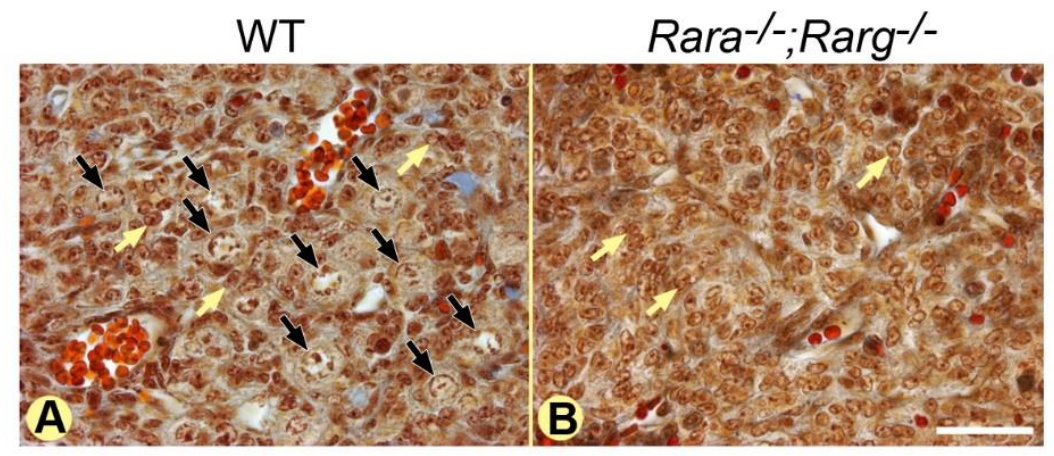

Figure 9. Meiotic oocytes are lacking in the ovary of some mouse mutant fetuses lacking both RARA and RARG. Representative histological sections of ovaries from ED18.5 WT and Rara ${ }^{-/} ;$Rarg $^{-1}$ female mice. See the main text for further details. Black and yellow arrows point to oocytes and ovarian somatic cells respectively. Trichrome stain. $B a r$ in $B=30 \mu \mathrm{m}(A$ and $B)$.

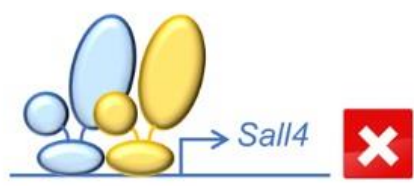

RXRA/RARG

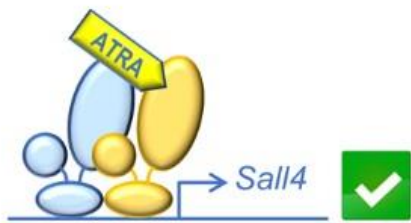

RXRA/RARG
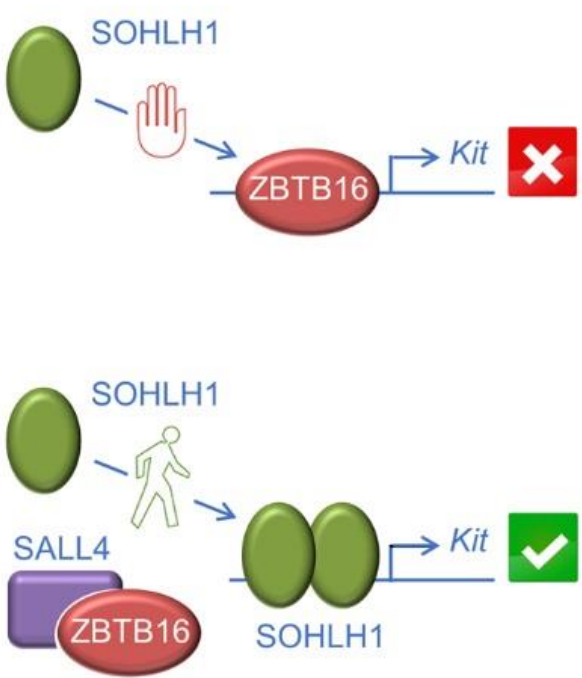

ZBTB16; KIT

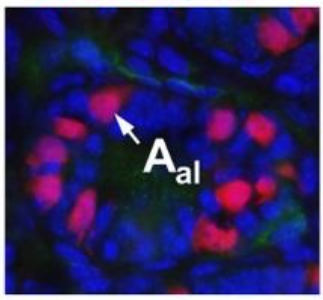

ZBTB16; KIT

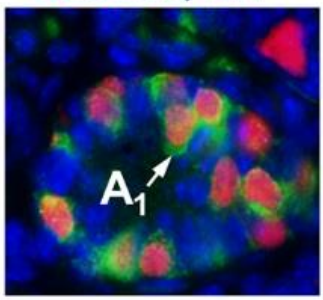

Figure 10. A model for the regulation of Kit expression by ATRA during the transition from $A_{a l}$ to $A_{1}$ spermatogonia. Upper panel: In $A_{a l}$ spermatogonia, ATRA is not available to activate the RXRA/RARG heterodimer and thus Sall 4 is transcribed at low levels. Transcription of Kit is also low because ZBTB16 is bound to its promoter (Filipponi et al, 2007). Lower panel: at the $A_{a l}-A_{1}$ transition, ATRA activates the RXRA/RARG heterodimer, which increases Sall4 expression. SALL4 in large amount can then sequesters ZBTB16, clearing thereby the Kit promoter and relieving the repression normally exerted by ZBTB16 (Hobbs et al., 2012). ATRA is also proposed to increase the level of SOHLH1, which can replace ZBTB16 on regulatory regions to increase Kit expression (Barrios et al., 2012). 